\title{
Non-Abelian Majorana Doublets in Time-Reversal-Invariant Topological Superconductors
}

\author{
Xiong-Jun Liu, ${ }^{1,2, *}$ Chris L. M. Wong, ${ }^{1}$ and K. T. Law ${ }^{1, \dagger}$ \\ ${ }^{1}$ Department of Physics, Hong Kong University of Science and Technology, \\ Clear Water Bay, Hong Kong, China \\ ${ }^{2}$ Institute for Advanced Study, Hong Kong University of Science and Technology, \\ Clear Water Bay, Hong Kong, China
}

(Received 19 June 2013; revised manuscript received 16 September 2013; published 29 April 2014)

\begin{abstract}
The study of non-Abelian Majorana zero modes advances our understanding of the fundamental physics in quantum matter and pushes the potential applications of such exotic states to topological quantum computation. It has been shown that in two-dimensional (2D) and 1D chiral superconductors, the isolated Majorana fermions obey non-Abelian statistics. However, Majorana modes in a $Z_{2}$ time-reversal-invariant (TRI) topological superconductor come in pairs due to Kramers's theorem. Therefore, braiding operations in TRI superconductors always exchange two pairs of Majoranas. In this work, we show interestingly that, due to the protection of time-reversal symmetry, non-Abelian statistics can be obtained in 1D TRI topological superconductors and may have advantages in applications to topological quantum computation. Furthermore, we unveil an intriguing phenomenon in the Josephson effect, that the periodicity of Josephson currents depends on the fermion parity of the superconducting state. This effect provides direct measurements of the topological qubit states in such 1D TRI superconductors.
\end{abstract}

DOI: 10.1103/PhysRevX.4.021018

\section{INTRODUCTION}

The search for exotic non-Abelian quasiparticles has been a focus of both theoretical and experimental studies in condensed matter physics, driven by both the exploration of the fundamental physics and the promising applications of such modes to a building block for a fault-tolerant topological quantum computer [1-7]. Following this pursuit, the topological superconductors have been brought to the forefront, for they host exotic zero-energy states known as Majorana fermions [8-16]. For a two-dimensional (2D) chiral $p+i p$-pairing state, which breaks time-reversal symmetry, one Majorana mode exists in each vortex core [2], and for the 1D $p$-wave case, such a state is located at each end of the system [4]. Because of the particle-hole symmetry, Majorana fermions in a topological superconductor are self-Hermitian modes that are identical to their own antiparticles. A complex fermion, whose quantum states span the physical space in the condensed-matter system, is formed by two Majoranas that can be located far away from each other. This property allows us to encode quantum information in the nonlocal fermionic states, which are topologically stable against local perturbations.

*phyliuxiongjun@gmail.com
pphlaw@ust.hk

Published by the American Physical Society under the terms of the Creative Commons Attribution 3.0 License. Further distribution of this work must maintain attribution to the author(s) and the published article's title, journal citation, and DOI.
Subject Areas: Condensed Matter Physics, Mesoscopics, Superconductivity
The existence of $2 n$ Majorana zero modes leads to $2^{n-1}$ fold ground-state degeneracy, and braiding two of such isolated modes in 2D or 1D superconductors transforms one state into another, which defines the non-Abelian statistics [3,15]. Remarkably, Majorana end states have been suggestively observed through tunneling measurements [17-19] in 1D effective $p$-wave superconductors obtained using heterostructures formed by semiconductor nanowire and $s$-wave superconductor [20-22].

Recently, a new class of topological superconductors with time-reversal symmetry, referred to as a DIIIsymmetry-class superconductor and classified by the $Z_{2}$ topological invariant [23-27], has attracted rapidly growing efforts [24-32]. Differently from chiral superconductors, in DIII-class superconductors, the zero modes come in pairs due to Kramers's theorem. Many interesting proposals have been studied to realize $Z_{2}$ time-reversal-invariant (TRI) Majorana quantum wires using the proximity effects of $d$ wave, $p$-wave, $s \pm$-wave, or conventional $s$-wave superconductors. It was shown that at each end of such a quantum wire are localized two Majorana fermions that form a Kramers doublet and are protected by time-reversal symmetry [30-38].

With the practicability in realization, a fundamental question is, can the DIII-class topological superconductor be applied to topological quantum computation? The puzzle arises from the fact that braiding the end states in a DIII-class 1D superconductor always exchanges Majorana Kramers pairs rather than isolated Majorana modes. While braiding two pairs of Majoranas in chiral 
topological superconductors yields Abelian operations, in this work, we show interestingly that braiding Majorana end states in DIII-class topological superconductors is nonAbelian due to the protection of time-reversal symmetry. We further unveil an intriguing phenomenon in the Josephson effect, that the periodicity of Josephson currents depends on the fermion parity of the superconducting state, which provides direct measurements of all topological qubit states in the DIII-class 1D superconductors.

The article is organized as follows. In Sec. II, we briefly introduce how to engineer the DIII-class 1D topological superconductor by inducing $p$-wave superconductivity in a conducting wire in proximity to a noncentrosymmetric superconductor. Then, in Sec. III, we turn to a detailed study of the non-Abelian Majorana doublets in the DIIIclass 1D topological superconductor. Section IV is devoted to investigating the Josephson effect, which shows an interesting strategy to read out topological qubit states in TRI superconductors. Finally, the conclusions are given in Sec. V.

\section{TOPOLOGICAL SUPERCONDUCTOR OF DIII CLASS BY PROXIMITY EFFECT}

Several interesting proposals have been considered to realize DIII-class 1D topological superconductors, including to use the proximity effects of $d$-wave, $p$-wave, $s \pm$ wave, and conventional $s$-wave superconductors [30-33]. Here, we briefly introduce how to engineer such a $Z_{2}$ topological superconductor by depositing a conducting quantum wire on a noncentrosymmetric superconductor thin film that can induce $s$-and $p$-wave pairings in the wire by the proximity effect $[39,40]$, as illustrated in Fig. 1 . The total Hamiltonian of the heterostructure system reads $H=H_{\mathrm{SC}}+H_{\text {wire }}+H_{t}$, where $H_{\mathrm{SC}}, H_{\text {wire }}$, and $H_{t}$ represent the Hamiltonians for the substrate superconductor, the conducting wire, and the tunneling at the interface, respectively. Because of the lack of inversion symmetry, a noncentrosymmetric superconductor has both $s$-wave and $p$-wave pairings [39]. For convenience, we denote the pairings in the substrate superconductor as $\Delta_{s}^{(0)}$ and $\Delta_{p}^{(0)}$, respectively. The Bogoliubov de Gennes Hamiltonian for the $2 \mathrm{D}$ noncentrosymmetric superconductor is given by

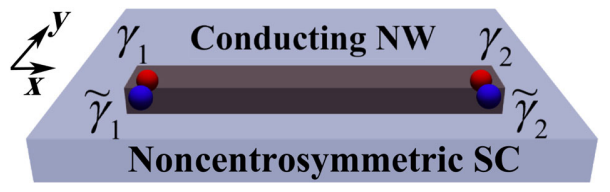

FIG. 1. The DIII-class 1D topological superconductor realized by depositing a conducting nanowire (NW) on the top of a noncentrosymmetric superconductor (SC). Both $s$ - and $p$-wave pairings can be induced in the conducting wire by tunneling couplings through the interface between the NW and the substrate superconductor.

$$
\begin{aligned}
H_{\mathrm{SC}}= & \sum_{k_{x}, k_{y}}\left[\epsilon_{0}\left(k_{x}, k_{y}\right) \tau_{z}+\alpha_{R}^{(0)} \sin k_{y} \sigma_{x}-\alpha_{R}^{(0)} \sin k_{x} \sigma_{y} \tau_{z}\right. \\
& \left.+\Delta_{p}^{(0)} \sin k_{y} \sigma_{z} \tau_{x}-\Delta_{p}^{(0)} \sin k_{x} \tau_{y}-\Delta_{s}^{(0)} \sigma_{y} \tau_{y}\right]
\end{aligned}
$$

where $\epsilon_{0}\left(k_{x}, k_{y}\right)=-2 t^{(0)}\left(\cos k_{x}+\cos k_{y}\right)-\mu^{(0)}$ is the normal dispersion relation, with $t^{(0)}$ the hopping coefficient in the superconductor; $\sigma_{j}$ and $\tau_{j}(j=x, y, z)$ are the Pauli matrices acting on the spin and Nambu spaces, respectively; $\alpha_{R}^{(0)}$ is the spin-orbit-coupling coefficient; and $\mu^{(0)}$ is the chemical potential. The pairing order parameters can be reorganized by $\hat{\Delta}=\left(\Delta_{s}^{(0)}+\mathbf{d} \cdot \sigma\right)\left(i \sigma_{y}\right)$, with the $\mathbf{d}$ vectors defined as $\mathbf{d}=\Delta_{p}^{(0)}\left(-\sin k_{y}, \sin k_{x}, 0\right)$.

A single-channel 1D conducting quantum wire, being put along the $x$ axis, can be described by the following Hamiltonian:

$$
H_{\text {wire }}=\sum_{k_{x}}\left(-2 t_{w} \cos k_{x}-\mu_{w}\right) \tau_{z}
$$

with $t_{w}$ the hopping coefficient and $\mu_{w}$ the chemical potential in the wire. It is noteworthy that the intrinsic spin-orbit interaction is not needed to reach the TRI topological superconducting phase, while the proximity effect can induce an effective spin-orbit interaction in the nanowire. Now, we give the tunneling Hamiltonian $H_{t}$ for the interface. For simplicity, we consider that at the interface, the coupling between the substrate superconductor and the nanowire is uniform, and thus the momentum $k_{x}$ is still a good quantum number. Then, the tunneling Hamiltonian can be written down as

$$
H_{t}=-t_{\perp} \sum_{k_{x}, \sigma} c_{\sigma}^{\dagger}\left(k_{x}\right) d_{i_{y 0}, \sigma}\left(k_{x}\right)+\text { H.c. }
$$

where $\sigma=\uparrow, \downarrow$ are the spin indices, $t_{\perp}$ denotes the tunneling coefficient between the nanowire and substrate superconductor, and $c_{\sigma}^{\dagger}, c_{\sigma}$ and $d_{\sigma}^{\dagger}, d_{\sigma}$ are the creation and annihilation operators of electrons for the quantum nanowire and the superconductor, respectively. The site number $i_{y 0}$ characterizes where the heterostructure is located on the $y$ axis in the noncentrosymmetric superconductor.

The induced superconductivity in the wire can be obtained by integrating out the degree of freedom of the superconductor substrate. We perform the integration in two steps. First, for the uniform noncentrosymmetric superconductor, we can determine its Green's function $G_{s}\left(k_{x}, i_{y 0}\right)$ with momentum $k_{x}$ and at the site $i_{y 0}$ below the nanowire by the standard recursive method [41]. Then, the coupling of the nanowire to the superconductor can be reduced to the coupling to the site $i_{y 0}$ below the wire and described by the Green's function $G_{s}\left(k_{x}, i_{y 0}\right)$. Integrating out the degree of freedom of the sites in the superconductor right below the nanowire yields a self-energy for 
the Green's function of the nanowire, which gives rise to the proximity effect. The effective Green's function of the nanowire takes the form

$$
G_{\text {wire }}\left(i \omega, k_{x}\right)=\frac{1}{i \omega-\epsilon_{w}\left(k_{x}\right) \tau_{z}-\Sigma(i \omega)},
$$

where $\epsilon_{w}=-2 t_{w} \cos k_{x}-\mu_{w}$ and the self-energy reads $\Sigma(i \omega)=t_{\perp}^{2} G_{s}\left(k_{x}, i_{y 0}\right)$. Finally, the spectral function is determined by

$$
A\left(\omega, k_{x}\right)=-\frac{1}{2 \pi} \operatorname{Im}\left\{\operatorname{Tr}\left[\tau_{z} G_{\text {wire }}\left(\omega+i 0^{+}, k_{x}\right)\right]\right\},
$$

with Im taking the imaginary part, $\operatorname{Tr}$ denoting the trace over the spin and Nambu spaces, and $0^{+}$a positive infinitesimal. The spectral function determines the bulk band structure, which is numerically shown in Fig. 2 with different chemical potentials of the nanowire. In particular, from the numerical results, we find that the nanowire is in the topologically nontrivial regime when $\left|\mu_{w}\right|<2\left|t_{w}\right|$ and $\left|\Delta_{p}^{(0)}\right|>\left|\Delta_{s}^{(0)}\right|$, which leads to the induced pairings in the wire $\left|\Delta_{p}\right|>\left|\Delta_{s}\right|$, while it is in the trivial regime when $\left|\Delta_{s}^{(0)}\right|>\left|\Delta_{p}^{(0)}\right|$ or $\left|\mu_{w}\right|>2\left|t_{w}\right|$ (i.e., the chemical potential is tuned out of the band of the wire). When tuning the
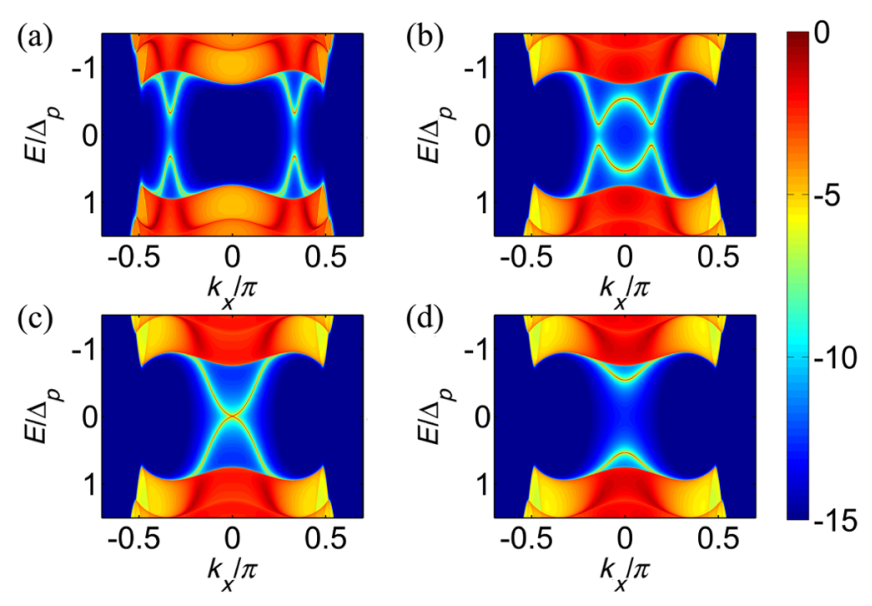

FIG. 2. The logarithmic plot of the spectral function for the nanowire or noncentrosymmetric superconductor heterostructure. The dotted yellow curves show the bulk band structure of the nanowire system. The solid red areas (in the upper and lower positions of each panel) represent the bulk states of the substrate superconductor. (a) Topological regime with the chemical potential $\mu_{w}$ set as $-2 t_{w}+5\left|\Delta_{p}^{(0)}\right|$ in the nanowire. In this regime, at each end of the wire are localized two Majorana zero modes (Fig. 3). (b) Topological regime with a reduced bulk gap by tuning $\mu_{w}=-2 t_{w}+\left|\Delta_{p}^{(0)}\right|$ close to the band bottom. (c) Critical point $\mu_{w}^{c}=-2 t_{w}$ for the topological phase transition with the bulk gap closed. (d) Trivial phase regime for the nanowire with $\mu=-2 t_{w}-\left|\Delta_{p}^{(0)}\right|$. Other parameters are taken that $t_{\perp}=0.5 t_{w}=$ $0.5 t^{(0)},\left|\Delta_{s}^{(0)}\right|=0.5\left|\Delta_{p}^{(0)}\right|$, and $\alpha_{R}^{(0)}=\left|\Delta_{p}^{(0)}\right|$. chemical potential down to the band bottom, the bulk gap in the nanowire is reduced and closes right at the bottom, implying the critical value of the chemical potential $\mu_{w}^{c}=$ $-2 t_{w}$ (similar results can be obtained around $2 t_{w}$, the top of the band) [Figs. 2(a)-2(c)]. In the topological regime at each end of the nanowire are localized two Majorana zero modes $\gamma_{j}$ and $\tilde{\gamma}_{j}(j=L, R)$ that form a Kramers's doublet, with their wave functions shown in Fig. 3. Further lowering the chemical potential reopens the bulk gap, and the system is driven into a trivial phase [Fig. 2(d)].

It is interesting that the phase diagram in the nanowire does not depend on parameter details of the couplings between the nanowire and the substrate superconductor, and for $\left|\Delta_{p}^{(0)}\right|>\left|\Delta_{s}^{(0)}\right|$, the topological regime in the nanowire can be obtained in a large parameter range that $-2 t_{w}<\mu_{w}<2 t_{w}$. This result enables a feasible way to engineer the DIII-class topological states in the experiment by tuning $\mu_{w}$ to be below or above the band bottom of the nanowire.

We note that the time-reversal symmetry is essential for the existence of the Majorana doublets in the topological phase. If time-reversal symmetry is broken, e.g., by introducing a Zeeman term $M_{z} \sigma_{z}$, the two Majorana modes at the same end will couple to each other and open a gap. On the other hand, while we consider here the DIII-class 1D topological superconductor through the proximity effect of noncentrosymmetric superconductors, the non-Abelian statistics predicted in this work are fundamental physics that are proposal independent and can also be studied with all other realistic setups for the 1D TRI topological superconductor, as proposed in recent works [30-38]. In particular, the recent proposal based on the proximity effect of $s$-wave conventional superconductors may be feasible for the experimental studies [33].

(a)

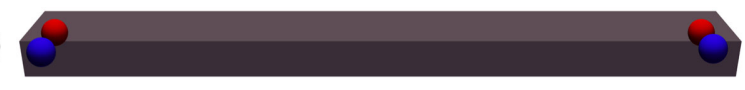

(b)
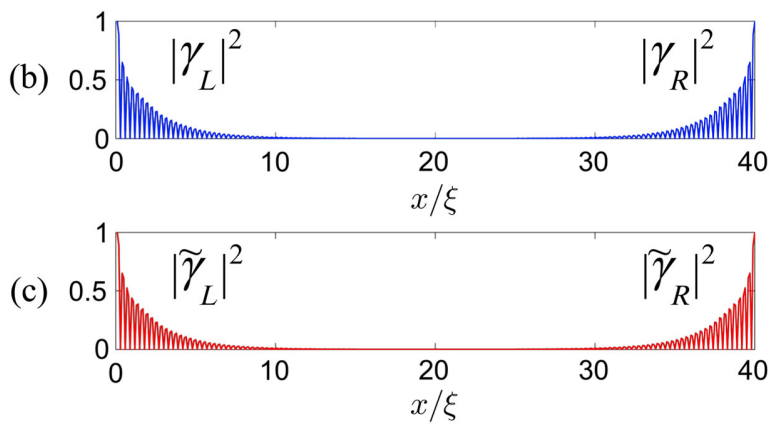

FIG. 3. (a) Two Majorana bound modes exist at each end of the nanowire in the topological regime with $\mu=-2 t_{w}+\left|\Delta_{p}^{(0)}\right|$, as considered in Fig. 2(b). (b),(c) The wave functions of two Majorana modes $\gamma_{L(R)}$ and $\tilde{\gamma}_{L(R)}$ at the same end have exactly the same spatial profile, with $\xi$ the coherence length in the nanowire. 


\section{NON-ABELIAN STATISTICS}

In this section, we show in detail that Majorana Kramers's doublets obey non-Abelian statistics due to the protection of time-reversal symmetry. In the previous section, we have demonstrated that for the topological phase, at each end of the $Z_{2}$ Majorana quantum wire are localized two Majorana modes $\gamma_{j}$ and $\tilde{\gamma}_{j}(j=1,2)$, transformed by a time-reversal operator that $\mathcal{T}^{-1} \gamma_{j} \mathcal{T}=$ $\tilde{\gamma}_{j}$ and $\mathcal{T}^{-1} \tilde{\gamma}_{j} \mathcal{T}=-\gamma_{j}$ [24]. To prove the non-Abelian statistics, we first show a new result that in a DIII-class topological superconductor at zero temperature, the fermion parity is conserved for each time-reversed sector of the system. With this result, we then get that braiding Majorana doublets can generically reduce to two independent processes of exchanging, respectively, two pairs of Majoranas belonging to two different time-reversed sectors, which leads to the symmetry-protected non-Abelian statistics.

\section{A. Fermi-parity conservation}

Fermion parity measures the even and odd numbers of the fermions in a quantum system. Note that the nonAbelian statistics are properties of the ground-state subspace of a topological superconductor. We only need to consider the superconductor at zero temperature, in which case no thermally excited quasiparticles exist and the superconductor is characterized as a condensate. The fermion number of a ground state can only vary by pairs due to the presence of a pairing gap, which leads to the fermion-parity conservation for the condensate. For the DIII-class 1D topological superconductor, we prove here a central result that by grouping all the fermionic modes into two sectors that are time-reversed partners of each other, the fermion parity is conserved for each sector of the condensate, not only for the entire system. It is trivial to know that this result is true if the DIII-class topological superconductor is composed of two decoupled copies (e.g., corresponding to the spin-up and spin-down, respectively) of 1D chiral $p$-wave superconductors. For the generic case, the proof is equivalent to showing that in a TRI Majorana quantum wire, which has four degenerate ground states $\left|n_{1} \tilde{n}_{1}\right\rangle\left(n_{1}, \tilde{n}_{1}=0,1\right)$, the four topological qubit states are decoupled from each other with the presence of finite TRI perturbations. (The change in the fermion parity for each sector of the condensate necessitates the transition between $|0 \tilde{1}\rangle$ and $|1 \tilde{0}\rangle$ or between $|0 \tilde{0}\rangle$ and $|1 \tilde{1}\rangle$.) The coupling Hamiltonian, assumed to depend on a manipulatable parameter $\lambda$, should take the generic TRI form $V(\lambda)=i E_{1}(\lambda)\left(\gamma_{1} \gamma_{2}-\tilde{\gamma}_{1} \tilde{\gamma}_{2}\right)+i E_{2}(\lambda)\left(\gamma_{1} \tilde{\gamma}_{2}-\gamma_{2} \tilde{\gamma}_{1}\right)$, which splits the two even-parity eigenstates $|0 \tilde{0}\rangle$ and $|1 \tilde{1}\rangle$ by an energy $E(\lambda)=2 \sqrt{E_{1}^{2}+E_{2}^{2}}$. Since $|1 \tilde{0}\rangle$ and $|0 \tilde{1}\rangle$ form a Kramers's doublet at an arbitrary $\lambda$ value, the transition between them is forbidden by time-reversal symmetry. Then, the fermion-parity conservation requires that the following adiabatic condition be satisfied in the manipulation: $\left|\left\langle 1 \tilde{1}\left|\dot{\lambda} \partial_{\lambda}\right| 0 \tilde{0}\right\rangle\right| \ll 2 E(\lambda)$, where $\dot{\lambda}=\partial \lambda / \partial t$. This criterion is followed by

$$
\tilde{R} \equiv \frac{1}{2 E(\lambda)}\left|\frac{\partial \lambda}{\partial t} \frac{\partial \theta}{\partial \lambda}\right| \ll 1, \quad \theta=\tan ^{-1} \frac{E_{1}}{E_{2}} .
$$

We show below that the above condition is generically satisfied under realistic conditions.

According to the the previous section, the proximity effect induces $p$-wave and $s$-wave superconducting pairings in the nanowire. The effective tight-binding Hamiltonian of the DIII-class Majorana nanowire in the generic case can be written as

$$
\begin{aligned}
H_{\mathrm{wire}}^{\mathrm{eff}}= & \sum_{\langle i, j\rangle, \sigma} t_{i j} c_{i \sigma}^{\dagger} c_{j \sigma}+\sum_{\langle i, j\rangle}\left(t_{i j}^{\mathrm{SO}} c_{i \uparrow}^{\dagger} c_{j \downarrow}+\text { H.c. }\right) \\
& +\sum_{\langle i, j\rangle}\left(\Delta_{i j}^{p} c_{i \uparrow} c_{j \uparrow}+\Delta_{i j}^{p *} c_{i \downarrow} c_{j \downarrow}+\text { H.c. }\right) \\
& +\sum_{j}\left(\Delta_{s} c_{j \uparrow} c_{j \downarrow}+\text { H.c. }\right)-\mu \sum_{j, \sigma} n_{j \sigma},
\end{aligned}
$$

where the hopping coefficients and the chemical potential are generically renormalized by the proximity effect, with the spin-conserved and the spin-orbit-coupled hopping terms satisfying $t_{i j}=t_{j i}=t$ and $t_{i j}^{\mathrm{SO}}=-t_{j i}^{\mathrm{SO}}=t_{\mathrm{SO}}$. For the case with uniform pairing orders, the parameters $\Delta_{s}$ and $\Delta_{p}$ can be taken as real. On the other hand, for the present 1D system, one can verify that the phases in the (spin-orbit) hopping coefficients can always be absorbed into electron operators. Therefore, below, we consider that all the parameters in $H_{\text {wire }}^{\text {eff }}$ are real numbers. Then, in terms of the electron operators, the Majorana bound modes take the following general forms:

$$
\begin{aligned}
\gamma_{1}= & \sum_{j}\left[u_{\uparrow}^{(1)}\left(x_{j}\right) c_{\uparrow}\left(x_{j}\right)+u_{\downarrow}^{(1)}\left(x_{j}\right) c_{\downarrow}\left(x_{j}\right)\right. \\
& \left.+u_{\uparrow}^{(1)}\left(x_{j}\right) c_{\uparrow}^{\dagger}\left(x_{j}\right)+u_{\downarrow}^{(1)}\left(x_{j}\right) c_{\downarrow}^{\dagger}\left(x_{j}\right)\right], \\
\gamma_{2}= & i \sum_{j}\left[u_{\uparrow}^{(2)}\left(x_{j}\right) c_{\uparrow}\left(x_{j}\right)+u_{\downarrow}^{(2)}\left(x_{j}\right) c_{\downarrow}\left(x_{j}\right)\right. \\
& \left.-u_{\uparrow}^{(2)}\left(x_{j}\right) c_{\uparrow}^{\dagger}\left(x_{j}\right)-u_{\downarrow}^{(2)}\left(x_{j}\right) c_{\downarrow}^{\dagger}\left(x_{j}\right)\right],
\end{aligned}
$$

and $\tilde{\gamma}_{j}=\mathcal{T} \gamma_{j} \mathcal{T}^{-1}$. The coupling energies between the Majorana modes at the left $\left(\gamma_{1}, \tilde{\gamma}_{1}\right)$ and right $\left(\gamma_{2}, \tilde{\gamma}_{2}\right)$ ends are calculated by $E_{1}=i\left\langle\gamma_{1}\left|H_{\text {wire }}^{\text {eff }}\right| \gamma_{2}\right\rangle=-i\left\langle\tilde{\gamma}_{1}\left|H_{\text {wire }}^{\text {eff }}\right| \tilde{\gamma}_{2}\right\rangle$ and $E_{2}=i\left\langle\gamma_{1}\left|H_{\text {wire }}^{\text {eff }}\right| \tilde{\gamma}_{2}\right\rangle=-i\left\langle\tilde{\gamma}_{1}\left|H_{\text {wire }}^{\text {eff }}\right| \gamma_{2}\right\rangle$.

It can be found that the coefficients $E_{1,2}$ are proportional to the overlapping integrals of the left- and right-end Majorana wave functions, which decay exponentially with the distance $d$ between the Majorana modes. Since $\gamma_{j}$ and $\tilde{\gamma}_{j}$ are connected by a $\mathcal{T}$ transformation, their wave functions 
have exactly the same spatial profile, which leads to the same exponential form of the coefficients $E_{1,2}(\lambda)=$ $\alpha_{1,2}(\lambda) e^{-d / \xi}$, with $\xi$ the coherence length in the nanowire. The prefactors $\alpha_{j}(\lambda)$ depend on the local couplings, i.e., the hopping terms and pairings in $H_{\text {wire }}^{\text {eff }}$, between electrons belonging to the same (for $j=1$ ) or different (for $j=2$ ) sectors of the time-reversal partners. For the realistic conditions, we consider that the chemical potential in the nanowire is far below the half-filling condition and thus the Fermi momentum satisfying $k_{F} a \ll 1$, and the coherence length (in the order of $1.0 \mu \mathrm{m}$ ) is typically much larger than the lattice constant $\xi \gg a(a \sim 0.5 \mathrm{~nm})$. Under these conditions, we can verify that to $E_{1,2}$, the contributions of the spin-orbit-coupling and $p$-wave-pairing terms in $H_{\text {wire }}^{\text {eff }}$ vanish, and we find (details can be found in the Appendix)

$$
\begin{gathered}
E_{1} \simeq t \sum_{\langle i, j\rangle \sigma} u_{\sigma}^{(1)}\left(x_{i}\right) u_{\sigma}^{(2)}\left(x_{j}\right), \\
E_{2} \simeq \Delta_{s} \sum_{\langle i, j\rangle \sigma} u_{\sigma}^{(1)}\left(x_{i}\right) u_{\sigma}^{(2)}\left(x_{j}\right) .
\end{gathered}
$$

Therefore, while the magnitudes of $E_{1,2}$ can vary with $d$ and the bulk gap, their ratio $E_{1} / E_{2}$ is nearly a constant, and we always have $\partial_{\lambda} \theta \approx 0$, which validates the adiabatic condition. The above results are consistent with the fact that when $\Delta_{s}=0$, the original Hamiltonian (7) can be block diagonalized, and then $E_{2} \equiv 0$. The adiabatic condition is clearly confirmed with the numerical results in Fig. 4. The fermion-parity conservation for each sector of the condensate shows that an isolated DIII-class 1D Majorana wire should stay in one of the four fermion-parity eigenstates germinated by nonlocal complex fermion operators $f_{j}$ and $\tilde{f}_{j}$, given that time-reversal symmetry is not broken. In particular, one can always prepare a nanowire initially in the ground state $|0 \tilde{0}\rangle$ or $|1 \tilde{1}\rangle$ by controlling the initial couplings $E_{1,2}(\lambda)$ and then manipulate the states adiabatically.

It is noteworthy that for a realistic system at finite temperature, the quasiparticle poisoning may exist, which can change fermion parity and lead to the decoherence of Majorana qubit states. At low temperature, the dominant effect in the quasiparticle poisoning comes from the singleelectron tunneling between the nanowire and the substrate superconductor [42]. The decoherence time in the chiral Majorana nanowires ranges from $10 \mathrm{~ns}$ to $0.1 \mathrm{~ms}$, depending on the parameter details [42]. We note that in realizing chiral topological superconductors, an external magnetic field is required to drive the system into the topological phase [20-22], and such a field suppresses the proximityinduced order parameter in the nanowires. Moreover, the topological gap in the nanowire depends on the ratio of the spin-orbit-coupling strength over the Zeeman energy and is further reduced by the magnetic field. As a result, in the (a)

(b)
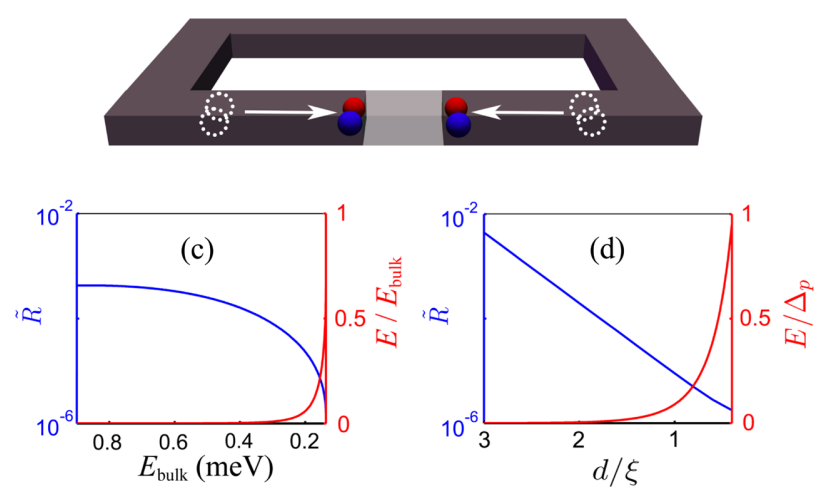

FIG. 4. Adiabatic condition and fermion-parity conservation for each sector of the superconductor. (a),(b) The couplings $E_{1,2}$ between Majorana modes are manipulated (a) by tuning the chemical that changes the bulk gap $\left(\lambda=E_{\text {bulk }}\right)$ in the nanowire and (b) by varying the length $(\lambda=d)$ of a trivial (gray) region that separates the two pairs of Majoranas. (c),(d) The energy splitting $E$ between $|0 \tilde{0}\rangle$ and $|1 \tilde{1}\rangle$ (red curves) and the ratio $\tilde{R}$ (blue curves), (c) as functions of $E_{\text {bulk }}$ and (d) versus the trivial region distance $d$. The parameters in the nanowire are taken such that the proximity-induced $p$-wave pairing $\Delta_{p}=1.0 \mathrm{meV}$, the $s$-wave pairing $\Delta_{s}=0.5 \mathrm{meV}$, and the spin-orbit-coupling energy $E_{\mathrm{SO}}=0.1 \mathrm{meV}$. In the numerical simulation, we assume that the coupling energy $E$ is tuned from 0 to $1.0 \mathrm{meV}$ in the time $1.0 \mu \mathrm{s}$. We also numerically confirm the adiabatic condition $\tilde{R} \ll 1$ with other different parameter regimes.

experiments on chiral Majorana nanowires, the topological gap is typically much smaller than the $s$-wave-pairing gap in the proximate superconductor. For the DIII-class nanowires, without suppression of the external magnetic field, the proximity-induced gap in the similar parameter regime is expected to be larger compared with that in chiral nanowires, which suggests a longer decoherence time in the DIII-class Majorana nanowires [30-33].

To ensure that the decoherence effect induced by quasiparticle poisoning does not lead to serious problems, one requires that the adiabatic manipulation time for Majorana modes should be much less than the decoherence time. For the DIII-class Majorana nanowires, the adiabatic time depends on the two characteristic time scales. One is determined by the bulk gap $\tau_{1}^{\text {ad }}=h / E_{g}$, and another $\tau_{2}^{\text {ad }}$ corresponds to the fermion-parity conservation for each time-reversal sector. The time scale $\tau_{1}^{\text {ad }}$ with an induced superconducting gap $0.1 \mathrm{meV}$ in the DIII-class wire is about $0.1 \mathrm{~ns}$, which is much less than the decoherence time. Furthermore, if using the parameter regime in Fig. 4, one can estimate that $\tau_{2}^{\text {ad }}<1.0 \mathrm{~ns}$. On the other hand, for the proposals considered in Refs. [30,31,33], the effective Hamiltonian has no $s$-wave-pairing order, and the time scale $\tau_{2}^{\text {ad }}$ then renders the magnitude of $\tau_{1}^{\text {ad }}$. These estimates 
imply that the adiabatic manipulation of Majorana modes may be reached in DIII-class 1D topological superconductors.

On the other hand, when a weak time-reversal-breaking term is present, e.g., in the presence of Zeeman couplings induced by a stray field, the decoherence effect may also result due to the couplings between qubit states with the same total fermion parity. Note that Majorana doublets in a DIII-class Majorana wire are of Ising type in the spin degree of freedom; thus, the time-reversal-breaking couplings can be induced by a stray field only along specific directions, depending on the concrete setup used in the experimental realization [35,37]. For the semiconductor nanowires with large Landé factors, e.g., the InAs wire that has a Landé factor $g \approx 20$ [22], one can verify that the resulting decoherence time is over $1.6 \mathrm{~ns}$ with a fluctuating stray field of strength $0.01 \mathrm{~T}$. This result implies that the decoherence effect is negligible when the field strength is several times less than $0.01 \mathrm{~T}$. In general, using semiconductor nanowires with small $g$ factors should be preferred in realizing the DIII-class topological superconductors, in which case the braiding operations can be stable against relatively stronger stray fields.

\section{B. Braiding statistics}

Note that braiding Majorana end modes is not well defined for a single 1D nanowire and, as first recognized by Alicea et al., the minimum setup for braiding requires a trijunction, e.g., a $T$ junction composed of two nanowire segments [15]. The braiding can be performed by transporting the Majorana zero modes following the steps as illustrated in Figs. 5(a)-5(d).

The fermion-parity conservation for each sector shown in the above subsection implies that the exchange of Majorana end modes in a DIII-class topological superconductor generically reduces to two independent processes of braiding Majoranas of two different sectors, respectively. The reason is because, first of all, braiding adiabatically the Majorana pairs, e.g., $\gamma_{1}, \tilde{\gamma}_{1}$ and $\gamma_{2}, \tilde{\gamma}_{2}$ in Fig. 5, does not affect the bulk states, which are gapped. Furthermore, assuming that other Majorana modes are
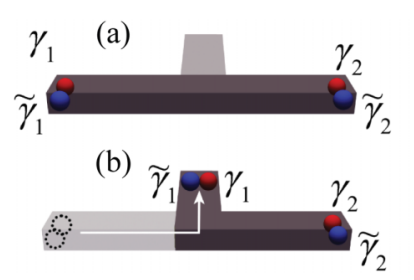
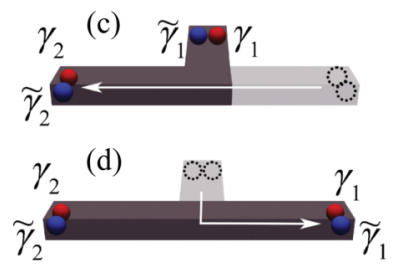

FIG. 5. (a)-(d) Braiding Majorana end modes through gating a $T$ junction following the study by Alicea $e t$ al. [15]. The dark (light) gray area of the nanowires depicts the topological (trivial) region, which can be controlled by tuning the chemical potential in the nanowire. The arrows depict the direction that the Majorana fermions are transported to in the braiding process. located far away from $\gamma_{1,2}$ and $\tilde{\gamma}_{1,2}$, the braiding evolves only the Majoranas that are exchanged. Finally, due to the fermion-parity conservation for the condensate, in the braiding, the qubit modes corresponding to $f_{1}$ and $\tilde{f}_{1}$ are decoupled and their evolution can be derived independently. By a detailed derivative, we show that after braiding, the topological qubit states evolve according to (see the Supplemental Material [43])

$$
\begin{aligned}
|1 \tilde{1}\rangle_{\text {final }} & =|1 \tilde{1}\rangle_{\text {initial }}, \\
|1 \tilde{0}\rangle_{\text {final }} & =i|1 \tilde{0}\rangle_{\text {initial }}, \\
|0 \tilde{1}\rangle_{\text {final }} & =-i|0 \tilde{1}\rangle_{\text {initial }} \\
|0 \tilde{0}\rangle_{\text {final }} & =|0 \tilde{0}\rangle_{\text {initial }}
\end{aligned}
$$

We therefore obtain the braiding matrix by $U_{12}(T, \tilde{T})=\exp \left[(\pi / 4) \gamma_{1} \gamma_{2}\right] \exp \left[(\pi / 4) \tilde{\gamma}_{1} \tilde{\gamma}_{2}\right]$, which is time-reversal invariant. This braiding operator can also be obtained in a different generic way. Because of the time-reversal symmetry, we have $U_{12}(T, \tilde{T})|1 \tilde{0}\rangle=e^{i \phi}|1 \tilde{0}\rangle$ and $U_{12}(T, \tilde{T})|0 \tilde{1}\rangle=e^{-i \phi}|0 \tilde{1}\rangle$, while $|1 \tilde{1}\rangle$ and $|0 \tilde{0}\rangle$ are unchanged. For the present DIII-class superconductor, one can show that $U_{12}^{4}=1$, which is followed by $\phi=0, \pi / 2$, or $\pi$. On the other hand, in the special situation that the DIIIclass topological superconductor is composed of two decoupled copies of 1D chiral $p$-wave superconductors, it can be derived straightforwardly that $\phi=\pi / 2$ [43]. Furthermore, the Hamiltonian of a generic DIII-class Majorana wire can be deformed continuously from the special one of two decoupled copies, while the phase factor $\phi$ only takes discrete values and cannot vary continuously. Therefore, the result $\phi=\pi / 2$ is valid for the generic case, completing the proof. Note that the oppositely handed braiding process of $U_{12}$ reads $U_{12}^{\dagger}(T, \tilde{T})=$ $\exp \left[-(\pi / 4) \gamma_{1} \gamma_{2}\right) \exp \left(-(\pi / 4) \tilde{\gamma}_{1} \tilde{\gamma}_{2}\right]$, which describes a process in which one first transports $\gamma_{2}$ and $\tilde{\gamma}_{2}$ to the end of the vertical wire, then transports the two modes $\gamma_{1}$ and $\tilde{\gamma}_{1}$ to the right-hand end, and finally, transports the two modes $\gamma_{2}$ and $\tilde{\gamma}_{2}$ to the left-hand end of the horizontal wire. The braiding matrix $U_{12}$ reflects that the two Majorana pairs $\gamma_{1}, \tilde{\gamma}_{1}$ and $\gamma_{2}, \tilde{\gamma}_{2}$ are braided independently, which leads to the symmetry-protected non-Abelian statistics as presented below.

We consider two DIII-class wire segments with eight Majorana modes $\gamma_{1, \ldots, 4}$ and $\tilde{\gamma}_{1, \ldots, 4}$ [Fig. 6(a)] that define four complex fermion modes by $f_{1}=\frac{1}{2}\left(\gamma_{1}+i \gamma_{2}\right)$, $f_{2}=\frac{1}{2}\left(\gamma_{3}+i \gamma_{4}\right)$, and $\tilde{f}_{1,2}=\mathcal{T}^{-1} f_{1,2} \mathcal{T}$. The Hilbert space of the four complex fermions is spanned by 16 qubit states $\left|n_{1} \tilde{n}_{1}\right\rangle_{L}\left|n_{2} \tilde{n}_{2}\right\rangle_{R}\left(n_{1,2}, \tilde{n}_{1,2}=0,1\right)$, where $L(R)$ represents the left (right) nanowire segment. If the initial state of the system is $|0 \tilde{0}\rangle_{L}|0 \tilde{0}\rangle_{R}$, for instance, by braiding the two pairs of Majoranas $\gamma_{2}, \tilde{\gamma}_{2}$ and $\gamma_{3}, \tilde{\gamma}_{3}$, we get straightforwardly 
(a)

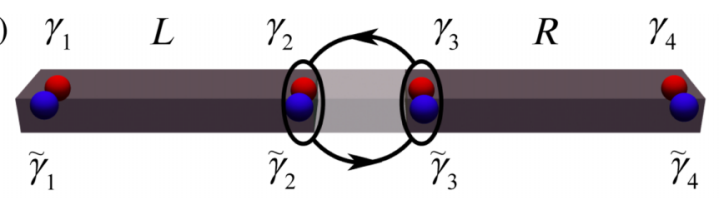

(b)

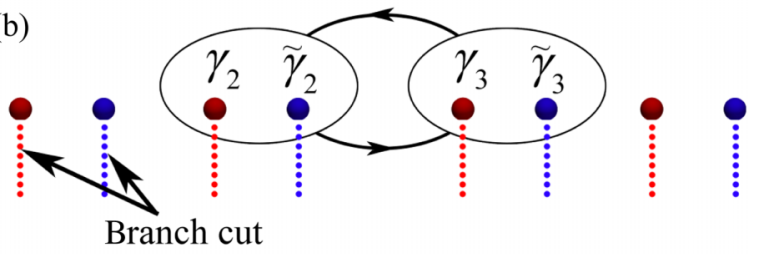

(c)

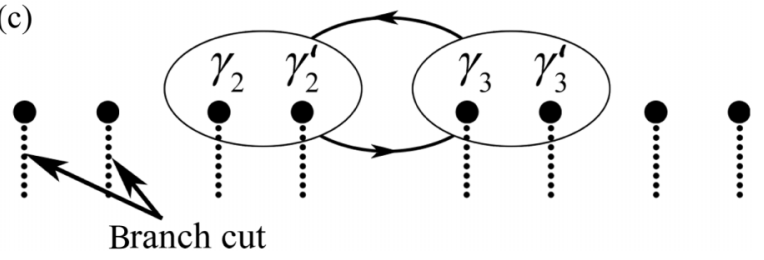

FIG. 6. Symmetry-protected non-Abelian statistics in a DIIIclass 1D topological superconductor. (a) Majorana end modes $\gamma_{2}$, $\tilde{\gamma}_{2}$ and $\gamma_{3}, \tilde{\gamma}_{3}$ are braided through similar processes to those shown in Fig. 5. (b) Braiding Majorana modes in a DIII-class superconductor are equivalent to two independent processes of exchanging $\gamma_{2}, \gamma_{3}$ and $\tilde{\gamma}_{2}, \tilde{\gamma}_{3}$, respectively. Majorana modes of one time-reversed sector do not experience the branch cuts of Majoranas belonging to another sector. In the depicted process, $\gamma_{2}$ $\left(\tilde{\gamma}_{2}\right)$ crosses only the branch cut of $\gamma_{3}\left(\tilde{\gamma}_{3}\right)$ and therefore acquires a minus sign after braiding. (c) In contrast, if braiding two Majorana pairs in a chiral superconductor, for the depicted process, $\gamma_{2}\left(\gamma_{2}{ }^{\prime}\right)$ crosses the branch cuts of both $\gamma_{3}$ and $\gamma_{3}{ }^{\prime}$, and then no sign change occurs for the Majorana operators after braiding [3]. Therefore, by braiding twice two Majorana pairs the system always returns to the original state.

$$
\begin{aligned}
U_{23}(T, \tilde{T})|0 \tilde{0}\rangle_{L}|0 \tilde{0}\rangle_{R}= & \frac{1}{2}\left(|0 \tilde{0}\rangle_{L}|0 \tilde{0}\rangle_{R}+|1 \tilde{1}\rangle_{L}|1 \tilde{1}\rangle_{R}\right. \\
& \left.+i|1 \tilde{0}\rangle_{L}|1 \tilde{0}\rangle_{R}-i|0 \tilde{1}\rangle_{L}|0 \tilde{1}\rangle_{R}\right)
\end{aligned}
$$

Here, the operator $U_{23}(T, \tilde{T})=\exp \left(\frac{\pi}{4} \gamma_{2} \gamma_{3}\right) \exp \left(\frac{\pi}{4} \tilde{\gamma}_{2} \tilde{\gamma}_{3}\right)$ takes the same form as $U_{12}$, while $\gamma_{2}, \tilde{\gamma}_{2}$ and $\gamma_{3}, \tilde{\gamma}_{3}$ are separated by a trivial region. Actually, the braiding operator is independent of the existence of $\gamma_{1,4}$ and $\tilde{\gamma}_{1,4}$. We can therefore fuse them by connecting the left-hand end of the $L$ wire and the right-hand end of the $R$ wire, and then $U_{23}(T, \tilde{T})$ is simply equivalent to exchanging two Majorana pairs for a single wire segment [15]. It is interesting that the above state is generically a fourparticle-entangled state, which shows the natural advantage in generating a multiparticle-entangled state using DIIIclass topological superconductors. Furthermore, a full braiding, i.e., braiding twice $\gamma_{2}, \tilde{\gamma}_{2}$ and $\gamma_{3}, \tilde{\gamma}_{3}$, yields the final state $|1 \tilde{1}\rangle_{L}|1 \tilde{1}\rangle_{R}$, which distinguishes from the initial state in that each copy of the $p$-wave superconductor changes fermion parity. After braiding four times the two pairs of Majoranas, the ground state returns to the original state. On the other hand, it is also straightforward to verify that $U_{12} U_{23} \neq U_{23} U_{12}$, implying the noncommutability of the braiding processes. These results demonstrate the non-Abelian statistics obeyed by Majorana doublets.

From the above discussion, we find that in the braiding, the Majorana modes $\gamma_{j}$ are unaffected by their time-reversal partners $\tilde{\gamma}_{j}$, which is an essential difference from the situation in exchanging two pairs of Majoranas in a chiral superconductor and makes the braiding operator in the TRI topological superconductor nontrivial. This property can be pictorialized by assigning branch cuts for the Majorana modes braided through the junction [15], as illustrated in Figs. 6(b) and 6(c). Majorana modes of one time-reversed sector do not experience the branch cuts of Majoranas belonging to another sector. When exchanging Majorana modes $\gamma_{2}, \gamma_{3}$ and $\tilde{\gamma}_{2}, \tilde{\gamma}_{3}$ in the DIII-class superconductor, $\gamma_{2}$ $\left(\tilde{\gamma}_{2}\right)$ crosses only the branch cut of $\gamma_{3}\left(\tilde{\gamma}_{3}\right)$ and therefore acquires a minus sign after braiding. In contrast, if braiding two Majorana pairs in a chiral superconductor, for the process in Fig. 6(c), $\gamma_{2}\left(\gamma_{2}^{\prime}\right)$ crosses the branch cuts of both $\gamma_{3}$ and $\gamma_{3}{ }^{\prime}$, and then no sign change occurs for the Majorana operators after braiding [3]. Therefore, a full braiding of two Majorana pairs always returns to the original state.

It is worthwhile to note that realizing a DIII-class superconductor applies no external magnetic field, which might be helpful to construct a realistic Majorana network to implement braiding operations. In comparison, for the chiral topological superconductor observed in a spin-orbitcoupled semiconductor nanowire using the $s$-wave superconducting proximity effect [20-22], the external magnetic field should be applied perpendicular to the spin-quantization axis by spin-orbit interaction, driving optimally the nanowire into the topological phase $[20,22,44]$. It is shown that for a network formed by multiple nanowire segments, such an optimal condition cannot be reached for all segments without inducing detrimental orbital effects, which creates further experimental challenges in braiding Majoranas [44]. It is clear that such an intrinsic difficulty is absent in the present DIIIclass TRI topological superconductor, and one might have more flexibility in constructing 2D and even 3D Majorana networks for topological quantum computation.

\section{JOSEPHSON EFFECT IN DIII-CLASS TOPOLOGICAL SUPERCONDUCTOR}

It is important to study how to detect the topological qubit states in a DIII-class Majorana quantum wire. The ground states of a single DIII-class Majorana quantum wire include two even- $(|0 \tilde{0}\rangle$ and $|1 \tilde{1}\rangle)$ and two odd- $(|0 \tilde{1}\rangle$ and $|1 \tilde{0}\rangle)$ parity eigenstates. In a chiral topological superconductor, the ground states of the same fermion parity are not distinguishable. On other hand, in the generic case, the two different time-reversal sectors do not correspond to 
different measurable good quantum numbers. Therefore, the two qubit states with the same total fermion parity, e.g., $|0 \tilde{1}\rangle$ and $|1 \tilde{0}\rangle$, cannot be distinguished via direct quantum-number measurements. However, according to the fermion-parity conservation shown in Sec. III A for each time-reversed sector of the condensate, in a 1D TRI topological superconductor, the two even- or odd-parity states are decoupled due to time-reversal symmetry, implying that such two states should be distinguishable. We show in this section that all four topological qubit states can be measured by the Josephson effect in DIII-class topological superconductors.

We consider a Josephson junction illustrated in Fig. 7(a) formed by DIII-class superconductor. As derived in the Appendix, the effective coupling Hamiltonian of the Josephson junction is given by

$$
\begin{aligned}
H_{\mathrm{eff}}(\phi)= & i \Gamma_{0} \cos \frac{\phi}{2}\left(\gamma_{L} \gamma_{R}-\tilde{\gamma}_{L} \tilde{\gamma}_{R}\right) \\
& +i \Gamma_{1} \sin \phi\left(\gamma_{L} \tilde{\gamma}_{L}-\gamma_{R} \tilde{\gamma}_{R}\right),
\end{aligned}
$$

where $\phi$ is the phase difference across the junction, and $L$ $(R)$ represents the left-hand (right-hand) lead of the junction. The $\Gamma_{0}$ term in $H_{\text {eff }}$ represents the first-order direct coupling between Majorana fermions at different junction leads. It can be seen that the direct coupling term is of $4 \pi$ periodicity, which can be understood in the following way. When the phase difference across the junction (a)

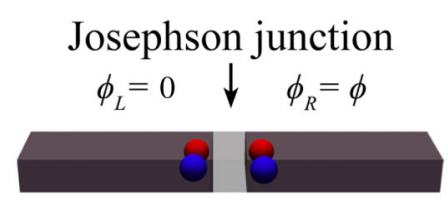

(c)

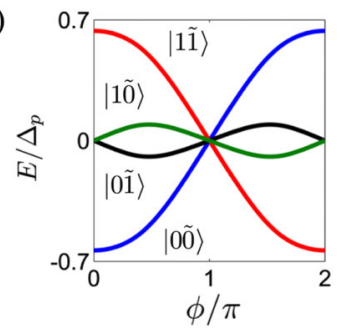

(b)

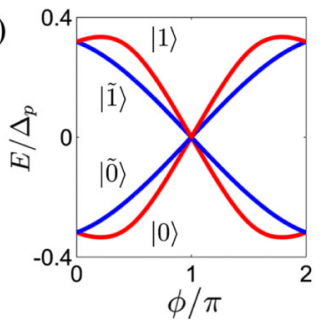

(d)

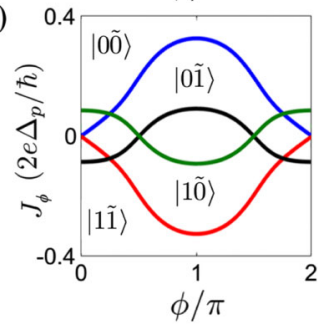

FIG. 7. Josephson measurement of the topological qubit states in a DIII-class 1D topological superconductor. (a) The sketch of a Josephson junction with phase difference $\phi$. (b) The singleparticle Andreev bound-state spectra versus the phase difference $\phi$. (c) The energy spectra of the four qubit states $\left|n_{1} \tilde{n}_{1}\right\rangle$ $\left(n_{1}, \tilde{n}_{1}=0,1\right)$ according to the results in (b). (d) The Josephson currents (in units of $2 e \Delta_{p} / \hbar$ ) for different topological qubit states. Parameters used in the numerical calculation are taken such that $\Delta_{p}=1.0 \mathrm{meV}, \Delta_{s}=0.25 \mathrm{meV}, E_{\mathrm{SO}}=0.1 \mathrm{meV}$, and the width of the junction $d=0.5 \xi$, and in the middle trivial (gray) region of the junction, the chemical potential is set to be at the band bottom. advances $2 \pi$, the Cooper-pair wave function changes $2 \pi$ across the junction, while for single-electron operators, the phase varies only $\pi$. This property implies that the periodic coupling coefficients also change the $\pi$ phase and thus reverse sign, leading to the $4 \pi$ periodicity of the direct coupling term. The $\Gamma_{1}$ term results from the second-order perturbation of the tunneling process, and this term vanishes if the $s$-wave pairing $\Delta_{s}=0$. The reason is because the couplings such as $i \gamma_{j} \tilde{\gamma}_{j}(j=L, R)$ break time-reversal symmetry, while the direct coupling between $\gamma_{j}$ and $\tilde{\gamma}_{j}$ does not experience the phase difference across the junction and should preserve time-reversal symmetry. Actually, a uniform pairing phase in one end of the junction can be removed by a constant gauge transformation. Therefore, the coupling between Majorana fermions at the same end can only be induced by electron tunneling and the minimum requirement is to consider the second-order tunneling process. In the second-order perturbation, $\gamma_{L}$ and $\tilde{\gamma}_{L}\left(\gamma_{R}\right.$ and $\left.\tilde{\gamma}_{R}\right)$ couple to the electron modes $c_{R}$ and $\tilde{c}_{R}$ in the right-hand end ( $c_{L}$ and $\tilde{c}_{L}$ in the left-hand end), respectively. When a nonzero $s$-wave pairing is present in the nanowires, the electrons $c_{L(R)}$ and $\tilde{c}_{L(R)}$ form a Cooper pair and condense. This process leads to the effective coupling between Majorana zero modes localized at the same end, with the coupling strength proportional to the $s$-wave order parameter. Finally, note that the system restores timereversal symmetry at $\phi=m \pi$, which explains why the $\Gamma_{1}$ term is proportional to $\sin \phi$ and has $2 \pi$ periodicity. All these properties have been confirmed with numerical results.

Redefining the Majorana bases by $\gamma_{1}^{\prime}=\gamma_{L}+\tilde{\gamma}_{R}$ and $\gamma_{2}^{\prime}=\gamma_{R}+\tilde{\gamma}_{L}$, we recast the above Hamiltonian into $H_{\text {eff }}=$ $i\left(\Gamma_{0} \cos \phi / 2+\Gamma_{1} \sin \phi\right) \gamma_{1}^{\prime} \gamma_{2}^{\prime}-i\left(\Gamma_{0} \cos \phi / 2-\Gamma_{1} \sin \phi\right) \tilde{\gamma}_{1}^{\prime} \tilde{\gamma}_{2}^{\prime}$. The Andreev bound-state spectra are obtained straight forwardly by

$$
\begin{aligned}
E_{n_{f^{\prime}}, \tilde{n}_{f^{\prime}}}(\phi)= & \left(\Gamma_{0} \cos \frac{\phi}{2}+\Gamma_{1} \sin \phi\right)\left(2 n_{f^{\prime}}-1\right) \\
& +\left(\Gamma_{0} \cos \frac{\phi}{2}-\Gamma_{1} \sin \phi\right)\left(2 n_{\tilde{f}^{\prime}}-1\right),
\end{aligned}
$$

which is shown numerically in Figs. 7(b) and 7(c). Here, $n_{f^{\prime}, \tilde{f}^{\prime}}$ are complex fermion-number operators for $f^{\prime}$ and $\tilde{f}^{\prime}$ modes, respectively. The Josephson currents are obtained by the slope of the Andreev bound-state spectra. In particular, we have that the Josephson currents $J_{\phi}^{\text {even }}=$ $\pm \frac{e}{\hbar} \Gamma_{0} \sin \frac{\phi}{2}$ for the even-parity states $|0 \tilde{0}\rangle$ and $|1 \tilde{1}\rangle$ and $J_{\phi}^{\text {odd }}= \pm \frac{e}{\hbar} \Gamma_{1} \cos \phi$ for the odd-parity states $|0 \tilde{1}\rangle$ and $|1 \tilde{0}\rangle$, respectively [Fig. 7(d)].

It is remarkable that the currents for odd-parity states are of $2 \pi$ periodicity, half of those for even-parity states [Fig. 7(d)]. This result reflects that $J_{\phi}^{\text {even }}$ is contributed from the direct Majorana coupling induced by first-order 
single-electron tunneling [4], while $J_{\phi}^{\text {odd }}$ is a consequence of the second-order tunneling process, which corresponds to the Cooper-pair tunneling. This nontrivial property is essentially different from the the Josephson physics with multiple Majorana end modes studied by Sticlet et al. in the BDI-class Majorana chains [45], where a multicopy version of the fractional Josephson effect with $4 \pi$ periodicity is investigated. The reason is because in a BDI-class topological superconductor, the time-reversal-symmetry operator $\mathcal{T}^{2}=1$ and the different copies of the superconductor are not related by time-reversal symmetry (nor by any other symmetry), while in the DIII-class topological superconductor, the two copies are related by $\mathcal{T}$ symmetry. The present result is also consistent with the fact that the timereversal symmetry is restored with $|0 \tilde{1}\rangle$ and $|1 \tilde{0}\rangle$, forming a Kramers's doublet at $\phi=m \pi$, which necessitates the $2 \pi$ periodicity in their spectra. Furthermore, the two qubit states with the same total parity (e.g., $|0 \tilde{0}\rangle$ and 1 1 $\rangle$ ) are distinguished by the direction of the currents. The qualitative difference in the Josephson currents implies that the four topological qubit states can be measured in the experiment.

\section{CONCLUSIONS}

In summary, we have shown that Majorana doublets obtained in the DIII-class 1D topological superconductors obey non-Abelian statistics, due to the protection of timereversal symmetry. The key results are that the fermion parity is conserved for each copy of the $Z_{2}$ TRI topological superconductor, and the exchange of Majorana end modes can generically reduce to two independent processes of braiding Majoranas of two different copies, respectively. These results lead to the symmetry-protected non-Abelian statistics for the Majorana doublets, and the braiding statistics are protected by time-reversal symmetry. Furthermore, we unveiled an intriguing phenomenon in the Josephson effect, that the periodicity of Josephson currents depends on the fermion parity of the 1D TRI topological superconductors. We found that this effect can provide direct measurements of the topological qubit states in the DIII-class Majorana quantum wires. Our results will motivate further studies in both theory and experiments on the braiding statistics and nontrivial Josephson effects in the wide classes of symmetry-protected topological superconductors.

\section{ACKNOWLEDGMENTS}

We appreciate the very helpful discussions with P. A. Lee, J. Alicea, L. Fu, Z.-X. Liu, A. Potter, Z.-C. Gu, M. Cheng, C. Wang, and X.G. Wen. The authors thank HKRGC for support through DAG12SC01, Grants No. 602813, No. 605512, and No. HKUST3/CRF/13G.

\section{APPENDIX}

\section{Fermi-parity conservation}

We consider a single Majorana quantum wire, which hosts four Majorana end modes denoted by $\gamma_{1,2}$ and $\tilde{\gamma}_{1,2}$ and is transformed via $\mathcal{T}^{-1} \gamma_{j} \mathcal{T}=\tilde{\gamma}_{j}$ and $\mathcal{T}^{-1} \tilde{\gamma}_{j} \mathcal{T}=-\gamma_{j}$. With the four Majorana states, we can define two nonlocal complex fermions by $f_{1}=\frac{1}{2}\left(\gamma_{1}+i \gamma_{2}\right)$ and $\tilde{f}_{1}=\frac{1}{2}\left(\tilde{\gamma}_{1}-i \tilde{\gamma}_{2}\right)$, which germinate four topological qubit states $\left|n_{1} \tilde{n}_{1}\right\rangle$ with $n_{1}, \tilde{n}_{1}=0,1$. Note that at zero temperature, the superconductor stays in the ground-state manifold. The proof of fermion-parity conservation for each sector of the condensate is equivalent to showing that the four topological qubit states $\left|n_{1} \tilde{n}_{1}\right\rangle$ are generically decoupled from each other in the presence of TRI perturbations.

Note that the coupling between the Majorana modes localized at the same end of the nanowire $\gamma_{j}$ and $\tilde{\gamma}_{j}$ breaks time-reversal symmetry. The coupling Hamiltonian in terms of Majorana end modes should take the following generic TRI form:

$V(\lambda)=i E_{1}(\lambda)\left(\gamma_{1} \gamma_{2}-\tilde{\gamma}_{1} \tilde{\gamma}_{2}\right)+i E_{2}(\lambda)\left(\gamma_{1} \tilde{\gamma}_{2}-\gamma_{2} \tilde{\gamma}_{1}\right)$,

where we assume that the couplings coefficients $E_{1,2}(\lambda)$ depend on an experimentally manipulatable parameter $\lambda$ (e.g., the bulk gap in the nanowire or the distance between the Majorana modes). The above Hamiltonian can be rewritten in the block-diagonal form with new Majorana bases such that

$$
V(\lambda)=i E(\lambda)\left[\gamma^{(1)} \gamma^{(2)}-\tilde{\gamma}^{(1)} \tilde{\gamma}^{(2)}\right],
$$

where $\gamma^{(1)}=\gamma_{1}, \quad \tilde{\gamma}^{(1)}=\tilde{\gamma}_{1}, \quad \gamma^{(2)}=\sin \theta \gamma_{2}+\cos \theta \tilde{\gamma}_{2}$, $\tilde{\gamma}^{(2)}=\sin \theta \tilde{\gamma}_{2}-\cos \theta \gamma_{2}$, and $E=\sqrt{E_{1}^{2}+E_{2}^{2}}$. The mixing angle $\theta$ is defined via $\tan \theta=E_{1} / E_{2}$. The complex fermions $f^{(1)}$ and $\tilde{f}^{(1)}$ in the eigenbasis are then defined by

$$
f^{(1)}=\frac{1}{2}\left[\gamma^{(1)}+i \gamma^{(2)}\right], \quad \tilde{f}^{(1)}=\frac{1}{2}\left[\tilde{\gamma}^{(1)}-i \tilde{\gamma}^{(2)}\right] .
$$

It is easy to know that the even-parity eigenstates $|0 \tilde{0}\rangle$ and $|1 \tilde{1}\rangle$ germinated by $f^{(1)}$ and $\tilde{f}^{(1)}$ acquire an energy splitting $2 E(\lambda)$, while the odd-parity states $|0 \tilde{1}\rangle$ and $|1 \tilde{0}\rangle$ are still degenerate due to time-reversal symmetry. To prove the fermion-parity conservation for each sector, we need to confirm that all four topological qubit states $\left|n_{1} \tilde{n}_{1}\right\rangle$ can evolve adiabatically when the coupling Hamiltonian $V(\lambda)$ varies with the parameter $\lambda$. Since $|1 \tilde{0}\rangle$ and $|0 \tilde{1}\rangle$ form a Kramers's doublet, the transition between them is forbidden by the time-reversal symmetry. Therefore, we only need to consider the adiabatic condition for the two even-parity states. The fermion-parity conservation for each sector is guaranteed when the following adiabatic condition is satisfied in the manipulation: 


$$
\left|\left\langle 1 \tilde{1}\left|\frac{\partial \lambda}{\partial t} \frac{\partial}{\partial \lambda}\right| 0 \tilde{0}\right\rangle\right| \ll 2|E(\lambda)|
$$

It should be noted that the adiabatic condition needs to be justified only in the presence of finite couplings. When $E(\lambda) \rightarrow 0$, the couplings between Majorana end modes vanish and then all the topological qubit states are automatically decoupled from each other. One can verify that

$$
\frac{\partial f^{(1)}}{\partial \lambda}=\frac{i}{2} \frac{\partial \theta}{\partial \lambda}\left(\cos \theta \gamma_{2}-\sin \theta \tilde{\gamma}_{2}\right)=\frac{1}{2} \frac{\partial \theta}{\partial \lambda}\left(\tilde{f}^{(1) \dagger}-\tilde{f}^{(1)}\right),
$$

$$
\frac{\partial \tilde{f}^{(1)}}{\partial \lambda}=-\frac{i}{2} \frac{\partial \theta}{\partial \lambda}\left(\cos \theta \tilde{\gamma}_{2}+\sin \theta \gamma_{2}\right)=-\frac{1}{2} \frac{\partial \theta}{\partial \lambda}\left(f^{(1) \dagger}-f^{(1)}\right) .
$$

With some calculation, one can show that in the above formulas, the derivatives of the bases $\gamma_{j}, \tilde{\gamma}_{j}$ with respect to $\lambda$ will not contribute to the left-hand side of Eq. (A4) and are therefore neglected. The condition (A4) then reads

$$
\left|\frac{\partial \lambda}{\partial t} \frac{\partial \theta}{\partial \lambda}\right| \ll 4|E(\lambda)|
$$

We show below that the above condition is generically satisfied in the realistic materials.

With the proximity-induced $p$-wave and $s$-wave superconducting pairings, the effective tight-binding Hamiltonian in the nanowire can be generically written as

$$
\begin{aligned}
H_{\mathrm{wire}}^{\mathrm{eff}}= & \sum_{\langle i, j\rangle, \sigma} t_{i j} c_{i \sigma}^{\dagger} c_{j \sigma}+\sum_{\langle i, j\rangle}\left(t_{i j}^{\mathrm{SO}} c_{i \uparrow}^{\dagger} c_{j \downarrow}+\text { H.c. }\right) \\
& +\sum_{\langle i, j\rangle}\left(\Delta_{i j}^{p} c_{i \uparrow} c_{j \uparrow}+\Delta_{i j}^{p *} c_{i \downarrow} c_{j \downarrow}+\text { H.c. }\right) \\
& +\sum_{j}\left(\Delta_{s} c_{j \uparrow} c_{j \downarrow}+\text { H.c. }\right) \\
& -\mu \sum_{j, \sigma} n_{j \sigma}+\sum_{j, \sigma} V_{j}^{\mathrm{dis}} n_{j \sigma},
\end{aligned}
$$

where the hopping coefficients and the chemical potential are generically renormalized by the proximity effect. Without loss of generality, in the above Hamiltonian, we have taken into account the spin-orbit interaction described by the $t_{i j}^{\mathrm{SO}}$ term and the random on-site disorder potential $V_{j}^{\text {dis }}$ with $\left\langle V_{j}^{\text {dis }}\right\rangle=0$. For the case with uniform pairing orders, the parameters $\Delta_{s}$ and $\Delta_{p}$ can be taken as real. On the other hand, for the present 1D system, one can verify that the phases in the (spin-orbit) hopping coefficients can always be absorbed into electron operators. Therefore, in the following study, we consider that all the parameters in $H_{\text {wire }}^{\text {eff }}$ are real numbers.

In the topological regime, at each end of the wire, we obtain two Majorana zero modes that are transformed to each other by a time-reversal operator. In terms of the electron operators, these bound modes take the form

$$
\begin{gathered}
\gamma_{1}=\sum_{j}\left[u_{\uparrow}^{(1)}\left(x_{j}\right) c_{\uparrow}\left(x_{j}\right)+u_{\downarrow}^{(1)}\left(x_{j}\right) c_{\downarrow}\left(x_{j}\right)\right. \\
\left.\quad+u_{\uparrow}^{(1) *}\left(x_{j}\right) c_{\uparrow}^{\dagger}\left(x_{j}\right)+u_{\downarrow}^{(1) *}\left(x_{j}\right) c_{\downarrow}^{\dagger}\left(x_{j}\right)\right], \\
\tilde{\gamma}_{1}=\sum_{j}\left[u_{\uparrow}^{(1) *}\left(x_{j}\right) c_{\downarrow}\left(x_{j}\right)-u_{\downarrow}^{(1) *}\left(x_{j}\right) c_{\uparrow}\left(x_{j}\right)\right. \\
\left.+u_{\uparrow}^{(1)}\left(x_{j}\right) c_{\downarrow}^{\dagger}\left(x_{j}\right)-u_{\downarrow}^{(1)}\left(x_{j}\right) c_{\uparrow}^{\dagger}\left(x_{j}\right)\right], \\
\gamma_{2}=i \sum_{j}\left[u_{\uparrow}^{(2)}\left(x_{j}\right) c_{\uparrow}\left(x_{j}\right)+u_{\downarrow}^{(2)}\left(x_{j}\right) c_{\downarrow}\left(x_{j}\right)\right. \\
\left.\quad-u_{\uparrow}^{(2) *}\left(x_{j}\right) c_{\uparrow}^{\dagger}\left(x_{j}\right)-u_{\downarrow}^{(2) *}\left(x_{j}\right) c_{\downarrow}^{\dagger}\left(x_{j}\right)\right], \\
\tilde{\gamma}_{2}=i \sum_{j}\left[u_{\uparrow}^{(2) *}\left(x_{j}\right) c_{\downarrow}\left(x_{j}\right)-u_{\downarrow}^{(2) *}\left(x_{j}\right) c_{\uparrow}\left(x_{j}\right)\right. \\
\left.\quad-u_{\uparrow}^{(2)}\left(x_{j}\right) c_{\downarrow}^{\dagger}\left(x_{j}\right)+u_{\downarrow}^{(2)}\left(x_{j}\right) c_{\uparrow}^{\dagger}\left(x_{j}\right)\right] .
\end{gathered}
$$

Note that the coefficients in $H_{\text {wire }}^{\text {eff }}$ are real, and we have that $u_{\uparrow, \downarrow}^{(1,2)}=u_{\uparrow, \downarrow}^{(1,2) *}$. The coupling energies between the Majorana modes at the left $\left(\gamma_{1}, \tilde{\gamma}_{1}\right)$ and right $\left(\gamma_{2}, \tilde{\gamma}_{2}\right)$ ends are calculated by $E_{1}=i\left\langle\gamma_{1}\left|H_{\text {wire }}^{\text {eff }}\right| \gamma_{2}\right\rangle=-i\left\langle\tilde{\gamma}_{1}\left|H_{\text {wire }}^{\text {eff }}\right| \tilde{\gamma}_{2}\right\rangle$ and $E_{2}=i\left\langle\gamma_{1}\left|H_{\text {wire }}^{\text {eff }}\right| \tilde{\gamma}_{2}\right\rangle=-i\left\langle\tilde{\gamma}_{1}\left|H_{\text {wire }}^{\text {eff }}\right| \gamma_{2}\right\rangle$. Using the relations

$$
\begin{aligned}
c_{j \uparrow} \simeq & u_{\uparrow}^{(1)}\left(x_{j}\right) \gamma_{1}-u_{\downarrow}^{(1)}\left(x_{j}\right) \tilde{\gamma}_{1}-i u_{\uparrow}^{(2)}\left(x_{j}\right) \gamma_{2} \\
& +i u_{\downarrow}^{(2)}\left(x_{j}\right) \tilde{\gamma}_{2}, \\
c_{j \downarrow} \simeq & u_{\downarrow}^{(1)}\left(x_{j}\right) \gamma_{1}+u_{\uparrow}^{(1)}\left(x_{j}\right) \tilde{\gamma}_{1}-i u_{\downarrow}^{(2)}\left(x_{j}\right) \gamma_{2}-i u_{\uparrow}^{(2)}\left(x_{j}\right) \tilde{\gamma}_{2},
\end{aligned}
$$

we obtain that

$$
\begin{aligned}
E_{1}= & \sum_{\langle i, j\rangle \sigma} t_{i j} u_{\sigma}^{(1)}\left(x_{i}\right) u_{\sigma}^{(2)}\left(x_{j}\right)+\sum_{\langle i, j\rangle \sigma} \Delta_{i j}^{p} u_{\sigma}^{(1)}\left(x_{i}\right) u_{\sigma}^{(2)}\left(x_{j}\right) \\
& +\sum_{\langle i, j\rangle} t_{i j}^{\mathrm{SO}}\left[u_{\uparrow}^{(1)}\left(x_{i}\right) u_{\downarrow}^{(2)}\left(x_{j}\right)+u_{\downarrow}^{(1)}\left(x_{j}\right) u_{\uparrow}^{(2)}\left(x_{i}\right)\right] \\
& +\sum_{j} \Delta_{s}\left[u_{\uparrow}^{(1)}\left(x_{j}\right) u_{\downarrow}^{(2)}\left(x_{j}\right)-u_{\downarrow}^{(1)}\left(x_{j}\right) u_{\uparrow}^{(2)}\left(x_{j}\right)\right] \\
& +\sum_{j, \sigma} V_{j}^{\mathrm{dis}} u_{\sigma}^{(1)}\left(x_{j}\right) u_{\sigma}^{(2)}\left(x_{j}\right),
\end{aligned}
$$




$$
\begin{aligned}
E_{2}= & \sum_{\langle i, j\rangle} t_{i j}\left[u_{\uparrow}^{(1)}\left(x_{i}\right) u_{\downarrow}^{(2)}\left(x_{j}\right)-u_{\downarrow}^{(1)}\left(x_{i}\right) u_{\uparrow}^{(2)}\left(x_{j}\right)\right] \\
& +\sum_{\langle i, j\rangle} \Delta_{i j}^{p}\left[u_{\uparrow}^{(1)}\left(x_{i}\right) u_{\downarrow}^{(2)}\left(x_{j}\right)-u_{\downarrow}^{(1)}\left(x_{i}\right) u_{\uparrow}^{(2)}\left(x_{j}\right)\right] \\
& +\sum_{\langle i, j\rangle} t_{i j}^{\mathrm{SO}}\left[u_{\uparrow}^{(1)}\left(x_{i}\right) u_{\uparrow}^{(2)}\left(x_{j}\right)-u_{\downarrow}^{(1)}\left(x_{j}\right) u_{\downarrow}^{(2)}\left(x_{i}\right)\right] \\
& +\sum_{j} \Delta_{s}\left[u_{\uparrow}^{(1)}\left(x_{j}\right) u_{\uparrow}^{(2)}\left(x_{j}\right)+u_{\downarrow}^{(1)}\left(x_{j}\right) u_{\downarrow}^{(2)}\left(x_{j}\right)\right] \\
& +\sum_{j, \sigma} V_{j}^{\mathrm{dis}}\left[u_{\uparrow}^{(1)}\left(x_{j}\right) u_{\downarrow}^{(2)}\left(x_{j}\right)-u_{\downarrow}^{(1)}\left(x_{j}\right) u_{\uparrow}^{(2)}\left(x_{j}\right)\right] .
\end{aligned}
$$

Note that $t_{i j}^{\mathrm{SO}}=-t_{j i}^{\mathrm{SO}}$ due to time-reversal symmetry, and for a uniform nanowire, we have that $\sum_{\langle i, j\rangle} u_{\uparrow}^{(1)}\left(x_{i}\right) u_{\downarrow}^{(2)}\left(x_{j}\right)=\sum_{\langle i, j\rangle} u_{\uparrow}^{(2)}\left(x_{i}\right) u_{\downarrow}^{(1)}\left(x_{j}\right) \quad$ and $\sum_{j} u_{\uparrow}^{(1)}\left(x_{j}\right) u_{\downarrow}^{(2)}\left(x_{j}\right)=\sum_{j} u_{\uparrow}^{(2)}\left(x_{j}\right) u_{\downarrow}^{(1)}\left(x_{j}\right)$. With these properties, we find that in $E_{1}$, the terms corresponding to $t_{i j}^{\mathrm{SO}}$ and $\Delta_{s}$ vanish, while in $E_{2}$, the terms for $t_{i j}, \Delta_{p}$, and $V_{j}^{\text {dis }}$ vanish. We then have

$$
\begin{aligned}
E_{1}= & \sum_{\langle i, j\rangle \sigma} t_{i j} u_{\sigma}^{(1)}\left(x_{i}\right) u_{\sigma}^{(2)}\left(x_{j}\right)+\sum_{\langle i, j\rangle \sigma} \Delta_{i j}^{p} u_{\sigma}^{(1)}\left(x_{i}\right) u_{\sigma}^{(2)}\left(x_{j}\right) \\
& +\sum_{j, \sigma} V_{j}^{\mathrm{dis}} u_{\sigma}^{(1)}\left(x_{j}\right) u_{\sigma}^{(2)}\left(x_{j}\right), \\
E_{2} & =\sum_{\langle i, j\rangle \sigma} t_{i j}^{\mathrm{SO}} u_{\sigma}^{(1)}\left(x_{i}\right) u_{\sigma}^{(2)}\left(x_{j}\right)+\sum_{j, \sigma} \Delta_{s} u_{\sigma}^{(1)}\left(x_{j}\right) u_{\sigma}^{(2)}\left(x_{j}\right) .
\end{aligned}
$$

The wave functions of Majorana bound modes decay exponentially as a function of the distance from the end of the nanowire, multiplying by an oscillatory function with the oscillating period equal to the Fermi wavelength in the nanowire. This property implies that $u_{\sigma}^{(1)} \propto$ $\sin \left(k_{F} x\right) e^{-x / \xi}$ and $u_{\sigma}^{(2)} \propto \sin e^{-(L-x) / \xi}$, where $\xi$ is the effective coherence length of the wire. In the realistic material, we consider that the chemical potential in the nanowire is far below the half-filling condition, and thus $k_{F} a \ll 1$. In this way, we have $u_{\sigma}^{(1)}\left(x_{j}\right) u_{\sigma}^{(2)}\left(x_{j}\right) \approx$ $u_{\sigma}^{(1)}\left(x_{j}\right) u_{\sigma}^{(2)}\left(x_{j \pm 1}\right) e^{\mp a / \xi}$. Furthermore, the coherence length (in the order of $1.0 \mu \mathrm{m}$ ) is typically much larger than the lattice constant $\xi \gg a(a \sim 0.5 \mathrm{~nm})$, and we can further approximate that $u_{\sigma}^{(1)}\left(x_{j}\right) u_{\sigma}^{(2)}\left(x_{j}\right) \approx u_{\sigma}^{(1)}\left(x_{j}\right) u_{\sigma}^{(2)}\left(x_{j \pm 1}\right)$. Bearing this result in mind, we get

$$
\begin{aligned}
E_{1}= & \sum_{\langle i, j\rangle \sigma} t_{i j} u_{\sigma}^{(1)}\left(x_{i}\right) u_{\sigma}^{(2)}\left(x_{j}\right)+\sum_{\langle i, j\rangle \sigma} \Delta_{i j}^{p} u_{\sigma}^{(1)}\left(x_{i}\right) u_{\sigma}^{(2)}\left(x_{j}\right) \\
& +\sum_{j, \sigma} V_{j}^{\mathrm{dis}} u_{\sigma}^{(1)}\left(x_{j}\right) u_{\sigma}^{(2)}\left(x_{j}\right)
\end{aligned}
$$

$$
E_{2}=\sum_{\langle i, j\rangle \sigma} t_{i j}^{\mathrm{SO}} u_{\sigma}^{(1)}\left(x_{i}\right) u_{\sigma}^{(2)}\left(x_{j}\right)+\sum_{\langle i, j\rangle \sigma} \Delta_{s} u_{\sigma}^{(1)}\left(x_{i}\right) u_{\sigma}^{(2)}\left(x_{j}\right)
$$

The spin-orbit hopping coefficient $t_{i j}^{\mathrm{SO}}=-t_{j i}^{\mathrm{SO}}$ and the $p$-wave pairing $\Delta_{i j}^{p}=-\Delta_{j i}^{p}$ are staggered parameters. In the limit that $k_{F} a \ll 1$ and $\xi \gg a$, the summation for such two terms in $E_{1}$ and $E_{2}$ also turns out to be 0 . On the other hand, the spin-conserved hopping is a constant, and we denote $t_{i j}=t_{j i}=t$. Finally, if the the random potential $V_{j}^{\text {dis }}$ with $\left\langle V_{j}^{\text {dis }}\right\rangle=0$ is distributed homogeneously in the nanowire, we expect that the last term in $E_{1}$ gives $V_{0} \sum_{j, \sigma} u_{\sigma}^{(1)}\left(x_{j}\right) u_{\sigma}^{(2)}\left(x_{j}\right)$, with the constant factor $V_{0}$ depending on the specific disorder profile and much less than the amplitude of the disorder potential. The couplings $E_{1,2}$ become

$$
\begin{gathered}
E_{1} \simeq\left(t+V_{0}\right) \sum_{\langle i, j\rangle \sigma} u_{\sigma}^{(1)}\left(x_{i}\right) u_{\sigma}^{(2)}\left(x_{j}\right), \\
E_{2} \simeq \Delta_{s} \sum_{\langle i, j\rangle \sigma} u_{\sigma}^{(1)}\left(x_{i}\right) u_{\sigma}^{(2)}\left(x_{j}\right) .
\end{gathered}
$$

From the above result, we find that $E_{2} / E_{1} \approx \Delta_{s} /\left(t+V_{0}\right)$, which is consistent with the fact that when $\Delta_{s}=0$, the original Hamiltonian (A8) can be block diagonalized, and then $E_{2} \equiv 0$. These results show that in the realistic nanowire materials, while the magnitudes of $E_{1,2}(\lambda)$ depend on $\lambda$, which determines the overlapping between the wave functions of Majorana bound modes at the left and right ends, their ratio is nearly a constant. Therefore, we always have

$$
\partial_{\lambda} \theta \approx 0
$$

which validates the adiabatic condition. The results in Eqs. (A21) and (A22) can be interpreted by an intuitive physical picture. Being proportional to the overlapping between the wave functions of Majorana bound modes at different ends, the coupling coefficients $E_{1,2}$ are exponential decaying functions of the nanowire length. Since the Majorana modes $\gamma_{j}$ and $\tilde{\gamma}_{j}$ are connected by a $\mathcal{T}$ transformation, their wave functions have exactly the same spatial profile, which leads to the same exponential form for the coefficients $E_{1,2}(\lambda)=\alpha_{1,2}(\lambda) e^{-d / \xi}$, with $d$ the distance between the left and right Majorana end modes. The prefactors $\alpha_{j}(\lambda)$ depend on the local couplings, i.e., the hopping coefficients and pairings between electrons belonging to the same (for $j=1$ ) or different (for $j=2$ ) sectors of the time-reversal partners. For the case with constant and homogeneous local couplings, we have that their ratio $\alpha_{1} / \alpha_{2}$ is proportional to the ratio of couplings between electrons of the same and different 

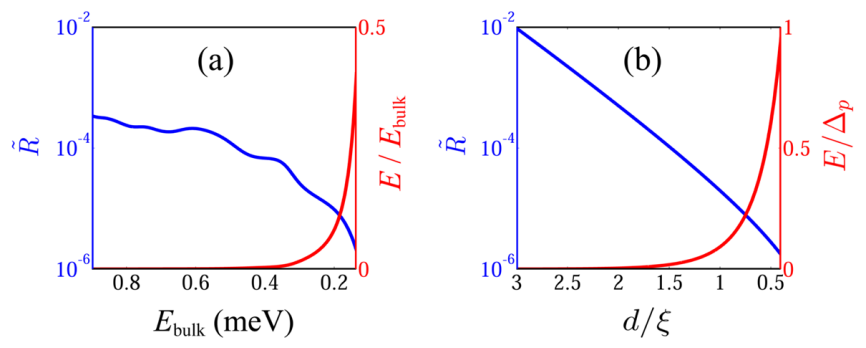

FIG. 8. Adiabatic condition and fermion-parity conservation for each sector of time-reversal partners in the presence of disorder scattering. The energy splitting $E$ between $|0 \tilde{0}\rangle$ and $|1 \tilde{1}\rangle$ (red curves) and the ratio $\tilde{R}$ (blue curves) versus (a) the bulk gap that varies by tuning the chemical potential and (b) the distance between the Majorana end modes. In the numerical simulation, the random on-site disorder potential is considered, with the potential amplitude $V_{\text {dis }} \sim 1.0 \mathrm{meV}$. Other parameters in the nanowire are taken such that $\Delta_{p}=1.0 \mathrm{meV}, \Delta_{s}=0.5 \mathrm{meV}$, and $E_{\mathrm{SO}}=0.1 \mathrm{meV}$. The coupling energy $E$ is tuned from 0 to $1.0 \mathrm{meV}$ within the time $1.0 \mu \mathrm{s}$.

sectors and is nearly a constant, justifying the adiabatic condition. The above derivative is clearly confirmed with numerical results in the realistic systems with the presence of random on-site disorder scattering, as shown in Fig. 8.

It is worthwhile to note that for a fixed parameter $\lambda$, the physics of the fermion-parity conservation for each sector can be easily understood in another way. For a DIII-class topological superconductor, the helical $p$-wave pairings occur between two electrons belonging to the same sector of the time-reversal partners. While the change by 1 in the fermion number of each sector conserves the total fermion parity of the system, it changes fermion parity for each sector and thus breaks a $p$-wave Cooper pair in each sector. This process costs finite energy and is completely suppressed by the $p$-wave-pairing gap at zero temperature, if $\Delta_{p}$ dominates over $\Delta_{s}$ and the time-reversal symmetry is not broken. The previous study in this section further proves this conservation law when the couplings between Majorana end modes are allowed and adjusted adiabatically. To simplify the notations in the further discussion, we relabel the block-diagonal Majorana modes $\gamma_{j}^{(1)}, \tilde{\gamma}_{j}^{(1)}$ as $\gamma_{j}$, $\tilde{\gamma}_{j}$. Accordingly, the diagonal complex fermion modes are redefined as $f_{j}, \tilde{f}_{j}$.

\section{Josephson effect in the DIII-class 1D topological superconductor}

Now, we study how to measure the topological qubit states with the Josephson effect. It has been predicted that in the chiral 1D topological superconductor, the Josephson current has $4 \pi$ periodicity [4], and the topological qubit states for a single wire $|0\rangle$ and $|1\rangle$ can be read out from the direction of Josephson currents in the junction $[8,15]$. In this section, we predict a novel phenomenon in the Josephson effect of the DIII-class 1D topological superconductor, which provides a feasible scheme to read out the topological qubit states in a TRI Majorana quantum wire.

\section{a. Effective coupling Hamiltonian}

We consider a Josephson junction formed by two Majorana nanowire ends with a phase difference $\phi=$ $\phi_{R}-\phi_{L}$, as illustrated in Fig. 9(a), and derive the effective coupling Hamiltonian for the Majorana zero modes localized at the left $(L)$ and right $(R)$ ends. The electron tunneling process in the junction is described by

$$
H_{T}=\Upsilon c_{L, N}^{\dagger} c_{R, 1}+\Upsilon \tilde{c}_{L, N}^{\dagger} \tilde{c}_{R, 1}+\text { H.c., }
$$

where $c_{L, N}, \tilde{c}_{L, N}$ and $c_{R, 1}, \tilde{c}_{R, 1}$ represent the electron operators for the $N$ th site at the left end and the first site at the right end of the junction, respectively, and $\Upsilon$ is the tunneling coefficient across the junction. The Majorana end modes can be generically expanded in terms of electron operators

$$
\begin{aligned}
& \gamma_{L}=\sum_{j}\left(u_{L, j} c_{L, j}+u_{L, j}^{*} c_{L, j}^{\dagger}\right), \\
& \tilde{\gamma}_{L}=\sum_{j}\left(\tilde{u}_{L, j} \tilde{c}_{L, j}+\tilde{u}_{L, j}^{*} \tilde{c}_{L, j}^{\dagger}\right),
\end{aligned}
$$

(a)

Josephson junction

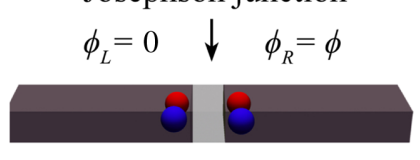

(c)

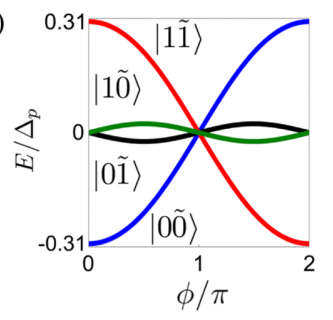

(b)

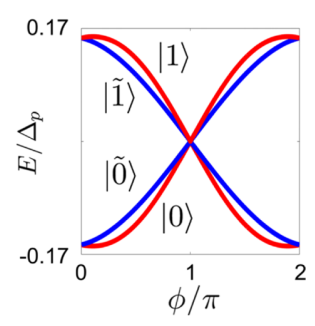

(d)

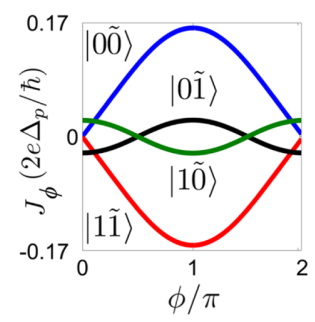

FIG. 9. Josephson effect in a DIII-class 1D topological superconductor with the inclusion of random disorder scattering. (a) The sketch of a Josephson junction with phase difference $\phi$. (b) The single-particle Andreev bound-state spectra versus the phase difference $\phi$. (c) The energy spectra of the four qubit states $\left|n_{1} \tilde{n}_{1}\right\rangle\left(n_{1}, \tilde{n}_{1}=0,1\right)$ according to the results in (b). (d) The Josephson currents (in units of $2 e \Delta_{p} / \hbar$ ) for different topological qubit states. In the numerical simulation, the amplitude of the random on-site disorder potential is set as $V_{\text {dis }} \sim 1.0 \mathrm{meV}$. Other parameters are taken such that $\Delta_{p}=1.0 \mathrm{meV}, \Delta_{s}=0.25 \mathrm{meV}$, $E_{\mathrm{SO}}=0.1 \mathrm{meV}$, and the width of the junction $d=0.75 \xi$, and in the middle trivial (gray) region of the junction, the chemical potential is set to be at the band bottom. 


$$
\begin{aligned}
& \gamma_{R}=\sum_{j}\left(u_{R, j} c_{R, j}+u_{R, j}^{*} c_{R, j}^{\dagger}\right), \\
& \tilde{\gamma}_{R}=\sum_{j}\left(\tilde{u}_{R, j} \tilde{c}_{R, j}+\tilde{u}_{R, j}^{*} \tilde{c}_{R, j}^{\dagger}\right),
\end{aligned}
$$

where $u_{L(R), j}=\tilde{u}_{L(R), j}^{*}$ if $\phi_{L(R)}=0$. Note that $c_{j}$ and $\tilde{c}_{j}$ represent electron operators of a general time-reversal pair at the $j$ th site, not necessarily corresponding to spin-up and spin-down, since the spin is not a good quantum number when spin-orbit coupling and $s$-wave order are present. From the above formulas, we can solve the electron operators in terms of Majorana and nonzero-energy Bogoliubov quasiparticle operators. Reexpressing the Bogoliubov quasiparticles in terms of electron operators, we can interpret $c_{L, N}, \tilde{c}_{L, N}$ and $c_{R, 1}, \tilde{c}_{R, 1}$ by

$$
c_{L, N}=u_{L, N}^{*} \gamma_{L}-\sum_{j=1}^{N} a_{L, j} c_{L, j}-\sum_{j=1}^{N} b_{L, j}^{*} c_{L, j}^{\dagger},
$$

$$
\begin{gathered}
\tilde{c}_{L, N}=\tilde{u}_{L, N}^{*} \gamma_{L}-\sum_{j=1}^{N} \tilde{a}_{L, j} \tilde{c}_{L, j}-\sum_{j=1}^{N} \tilde{b}_{L, j}^{*} \tilde{c}_{L, j}^{\dagger}, \\
c_{R, 1}=u_{R, 1}^{*} \gamma_{R}-\sum_{j=1}^{N} a_{R, j} c_{R, j}-\sum_{j=1}^{N} b_{R, j}^{*} c_{R, j}^{\dagger}, \\
\tilde{c}_{R, 1}=\tilde{u}_{R, 1}^{*} \gamma_{R}-\sum_{j=1}^{N} \tilde{a}_{R, j} \tilde{c}_{R, j}-\sum_{j=1}^{N} \tilde{b}_{R, j}^{*} \tilde{c}_{R, j}^{\dagger},
\end{gathered}
$$

with a constant normalization factor neglected. Here, $a_{L(R), j}, \tilde{a}_{L(R), j}$ and $b_{L(R), j}, \tilde{b}_{L(R), j}$ are expansion coefficients, originated from the quasiparticle operators other than the corresponding Majorana mode. Substituting these results into the tunneling Hamiltonian $H_{T}$ yields that

$$
\begin{aligned}
H_{T}= & \Upsilon\left(u_{L, N} \gamma_{L}-\sum_{j=1}^{N} a_{L, j}^{*} c_{L, j}^{\dagger}-\sum_{j=1}^{N} b_{L, j} c_{L, j}\right)\left(u_{R, 1}^{*} \gamma_{R}-\sum_{j=1}^{N} a_{R, j} c_{R, j}-\sum_{j=1}^{N} b_{R, j}^{*} c_{R, j}^{\dagger}\right) \\
& +\Upsilon\left(\tilde{u}_{L, N} \tilde{\gamma}_{L}-\sum_{j=1}^{N} \tilde{a}_{L, j}^{*} \tilde{c}_{L, j}^{\dagger}-\sum_{j=1}^{N} \tilde{b}_{L, j} \tilde{c}_{L, j}\right)\left(\tilde{u}_{R, 1}^{*} \tilde{\gamma}_{R}-\sum_{j=1}^{N} \tilde{a}_{R, j} \tilde{c}_{R, j}-\sum_{j=1}^{N} \tilde{b}_{R, j}^{*} \tilde{c}_{R, j}^{\dagger}\right)+\text { H.c. } \\
\approx & H^{(0)}+H^{(1)},
\end{aligned}
$$

where

$$
\begin{aligned}
H^{(0)}= & \Upsilon u_{L, N} u_{R, 1}^{*} \gamma_{L} \gamma_{R}+\Upsilon \tilde{u}_{L, N} \tilde{u}_{R, 1}^{*} \tilde{\gamma}_{L} \tilde{\gamma}_{R}+\text { H.c. } \\
H^{(1)}= & -\Upsilon u_{L, N} \gamma_{L}\left(\sum_{j=1}^{N} a_{R, j} c_{R, j}+\sum_{j=1}^{N} b_{R, j}^{*} c_{R, j}^{\dagger}\right)-\Upsilon u_{R, 1} \gamma_{R}\left(\sum_{j=1}^{N} a_{L, j} c_{L, j}+\sum_{j=1}^{N} b_{L, j}^{*} c_{L, j}^{\dagger}\right) \\
& -\Upsilon \tilde{u}_{L, N} \tilde{\gamma}_{L}\left(\sum_{j=1}^{N} \tilde{a}_{R, j} \tilde{c}_{R, j}+\sum_{j=1}^{N} \tilde{b}_{R, j}^{*} \tilde{c}_{R, j}^{\dagger}\right)-\Upsilon \tilde{u}_{R, 1} \tilde{\gamma}_{R}\left(\sum_{j=1}^{N} \tilde{a}_{L, j} \tilde{c}_{L, j}+\sum_{j=1}^{N} \tilde{b}_{L, j}^{*} \tilde{c}_{L, j}^{\dagger}\right)+\text { H.c. }
\end{aligned}
$$

In the second equation of the formula (A31), we have neglected the higher-order irrelevant terms. The term $H^{(0)}$ represents the direct coupling between Majorana modes at different junction ends, which gives the first term of the effective Hamiltonian $H_{\text {eff }}$ in the main text. This result can be seen by noticing that

$$
\begin{gathered}
u_{L, N}=i\left|u_{L, N}\right| e^{i \phi_{L} / 2}, \quad u_{R, 1}=\left|u_{R, 1}\right| e^{i \phi_{R} / 2}, \\
\tilde{u}_{L, N}=-i\left|\tilde{u}_{L, N}\right| e^{i \phi_{L} / 2}, \quad \tilde{u}_{R, 1}=\left|\tilde{u}_{R, 1}\right| e^{i \phi_{R} / 2},
\end{gathered}
$$

with which we can recast $H^{(0)}$ into

$$
\begin{aligned}
H^{(0)} & =i \Gamma_{0} \cos \frac{\phi}{2}\left(\gamma_{L} \gamma_{R}-\tilde{\gamma}_{L} \tilde{\gamma}_{R}\right), \\
\Gamma_{0} & =2 \Upsilon\left|u_{L, N} u_{R, 1}\right| .
\end{aligned}
$$

On the other hand, for $H^{(1)}$, we shall calculate up to the second-order perturbation, which is responsible for the second term of $H_{\text {eff }}$ in the main text. From $H^{(1)}$, we know that Majorana modes at one end (e.g., the left end) also couple to the electron modes at another end (the right end). In the second-order perturbation, $\gamma_{L}$ and $\tilde{\gamma}_{L}\left(\gamma_{R}\right.$ and $\left.\tilde{\gamma}_{R}\right)$ couple to $c_{R}$ and $\tilde{c}_{R}\left(c_{L}\right.$ and $\left.\tilde{c}_{L}\right)$, respectively. When a nonzero $s$-wave pairing is present in the quantum wires, the electrons $c_{L(R)}$ and $\tilde{c}_{L(R)}$ form a Cooper pair and condense. 
This process leads to an effective coupling between Majorana zero modes localized at the same end. Therefore, up to the second-order perturbation in the tunneling process, we obtain that

$$
\begin{aligned}
H_{\mathrm{eff}}^{(1)}= & \frac{1}{2} \Upsilon^{2} u_{L, N} \tilde{u}_{L, N} \gamma_{L} \tilde{\gamma}_{L}\left[\sum_{j=1}^{N} a_{R, j} \tilde{a}_{R, j} \int d \tau\left\langle T_{\tau} c_{R, j}(\tau) \tilde{c}_{R, j}(0)\right\rangle+\sum_{j=1}^{N} b_{R, j}^{*} \tilde{b}_{R, j}^{*} \int d \tau\left\langle T_{\tau} c_{R, j}^{\dagger}(\tau) \tilde{c}_{R, j}^{\dagger}(0)\right\rangle\right] \\
& +\frac{1}{2} \Upsilon^{2} u_{R, 1} \tilde{u}_{R, 1} \gamma_{R} \tilde{\gamma}_{R}\left[\sum_{j=1}^{N} a_{L, j} \tilde{a}_{L, j} \int d \tau\left\langle T_{\tau} c_{L, j}(\tau) \tilde{c}_{L, j}(0)\right\rangle+\sum_{j=1}^{N} b_{L, j}^{*} \tilde{b}_{L, j}^{*} \int d \tau\left\langle c_{L, j}^{\dagger}(\tau) \tilde{c}_{L, j}^{\dagger}(0)\right\rangle\right]+\text { H.c. }
\end{aligned}
$$

Here, $\int d \tau\left\langle T_{\tau} \cdots\right\rangle$ represents a time-ordered integral. Assuming that the superconducting pairings are uniform in the Majorana nanowires, we obtain from the above formula that

$$
\begin{aligned}
H_{\mathrm{eff}}^{(1)}= & \frac{1}{2} \Upsilon^{2} u_{L, N} \tilde{u}_{L, N} \gamma_{L} \tilde{\gamma}_{L}\left[\sum_{j=1}^{N} a_{R, j} \tilde{a}_{R, j} \sum_{k} \frac{\Delta_{s, R}^{*}}{\mathcal{E}_{R}^{2}\left(\Delta_{s, R} ; \Delta_{p, R} ; k\right)}-\sum_{j=1}^{N} b_{R, j}^{*} \tilde{b}_{R, j}^{*} \sum_{k} \frac{\Delta_{s, R}}{\mathcal{E}_{R}^{2}\left(\Delta_{s, R} ; \Delta_{p, R} ; k\right)}\right] \\
& +\frac{1}{2} \Upsilon^{2} u_{R, 1} \tilde{u}_{R, 1} \gamma_{R} \tilde{\gamma}_{R}\left[\sum_{j=1}^{N} a_{L, j} \tilde{a}_{L, j} \sum_{k} \frac{\Delta_{s, L}^{*}}{\mathcal{E}_{L}^{2}\left(\Delta_{s, L} ; \Delta_{p, L} ; k\right)}-\sum_{j=1}^{N} b_{L, j}^{*} \tilde{b}_{L, j}^{*} \sum_{k} \frac{\Delta_{s, L}}{\mathcal{E}_{L}^{2}\left(\Delta_{s, L} ; \Delta_{p, L} ; k\right)}\right]+\text { H.c. } \\
= & i \Upsilon_{L}(\phi) \gamma_{L} \tilde{\gamma}_{L}+i \tilde{\Upsilon}_{R}(\phi) \gamma_{R} \tilde{\gamma}_{R},
\end{aligned}
$$

with $\mathcal{E}_{L(R)}\left(\Delta_{s, R} ; \Delta_{p, R} ; k\right)$ the bulk excitation spectra in the left (for $L$ ) and right (for $R$ ) wires of the junction, respectively. The coupling coefficients read

$$
\begin{aligned}
\Upsilon_{L(R)}(\phi)= & -i \frac{1}{2} \Upsilon^{2} u_{L(R), N / 1} \tilde{u}_{L(R), N / 1}\left[\sum_{j=1}^{N} a_{R(L), j} \tilde{a}_{R(L), j} \sum_{k} \frac{\Delta_{s, R(L)}^{*}}{\mathcal{E}_{R(L)}^{2}\left(\Delta_{S, R(L)} ; \Delta_{p, R(L)} ; k\right)}\right. \\
& \left.-\sum_{j=1}^{N} b_{R(L), j}^{*} \tilde{b}_{R(L), j}^{*} \sum_{k} \frac{\Delta_{s, R(L)}}{\mathcal{E}_{R(L)}^{2}\left(\Delta_{s, R(L)} ; \Delta_{p, R(L)} ; k\right)}\right]- \text { c.c. }
\end{aligned}
$$

With the relations obtained in Eqs. (A27)-(A30), we have that $a_{R(L), j} \tilde{a}_{R(L), j}=\left|a_{R(L), j} \tilde{a}_{R(L), j}\right| \quad$ and $\quad b_{R(L), j} \tilde{b}_{R(L), j}=$ $\left|b_{R(L), j} \tilde{b}_{R(L), j}\right| e^{i 2 \phi_{R(L)}}$. Together with the results in

Eq. (A32), we can simplify $\Upsilon_{L(R)}(\phi)$ to be

$$
\begin{aligned}
& \Upsilon_{L}(\phi)=-i \Gamma_{1} e^{i \phi}-\text { c.c. }=\Gamma_{1} \sin \phi, \\
& \Upsilon_{R}(\phi)=-i \Gamma_{1} e^{-i \phi}-\text { c.c. }=-\Gamma_{1} \sin \phi,
\end{aligned}
$$

and the effective coupling Hamiltonian for Majorana fermions at the same end takes the following form:

$$
H_{\mathrm{eff}}^{(1)}=i \Gamma_{1} \sin \phi\left(\gamma_{L} \tilde{\gamma}_{L}-\gamma_{R} \tilde{\gamma}_{R}\right)
$$

The coupling constant $\Gamma_{1}$ is calculated by

$$
\Gamma_{1}=\Upsilon^{2}\left|u_{L, N} \tilde{u}_{L, N}\right|\left[\sum_{j=1}^{N}\left|a_{R, j} \tilde{a}_{R, j}\right| \sum_{k} \frac{\left|\Delta_{s, R}\right|}{\mathcal{E}_{R}^{2}\left(\Delta_{s, R} ; \Delta_{p, R} ; k\right)}-\sum_{j=1}^{N}\left|b_{R, j}^{*} \tilde{b}_{R, j}^{*}\right| \sum_{k} \frac{\left|\Delta_{s, R}\right|}{\mathcal{E}_{R}^{2}\left(\Delta_{s, R} ; \Delta_{p, R} ; k\right)}\right] .
$$

We have assumed the uniformity of the parameters in the left and right wires of the junction such that $\left|\Delta_{S, L}\right|=\left|\Delta_{s, R}\right|$ and $\left|\Delta_{p, L}\right|=\left|\Delta_{p, R}\right|$, and therefore $\left|u_{L, N} \tilde{u}_{L, N}\right|=\left|u_{R, 1} \tilde{u}_{R, 1}\right|$ and $\mathcal{E}_{L}\left(\Delta_{s, L} ; \Delta_{p, L} ; k\right)=\mathcal{E}_{R}\left(\Delta_{s, R} ; \Delta_{p, R} ; k\right)$. We note that this condition is typically satisfied in the realistic systems. It is clear that the $\Gamma_{1}$ term vanishes when the $s$-wave pairing $\Delta_{s, L(R)}$ is absent in the wires.

To this end, we combine $H^{(0)}$ and $H_{\text {eff }}^{(1)}$ to finally reach the effective Hamiltonian for a Josephson junction formed by DIII-class topological superconductors that

$$
\begin{aligned}
H_{\mathrm{eff}}(\phi)= & i \Gamma_{0} \cos \frac{\phi}{2}\left(\gamma_{L} \gamma_{R}-\tilde{\gamma}_{L} \tilde{\gamma}_{R}\right) \\
& +i \Gamma_{1} \sin \phi\left(\gamma_{L} \tilde{\gamma}_{L}-\gamma_{R} \tilde{\gamma}_{R}\right)
\end{aligned}
$$

Note that if treating $\phi$ as a fixed parameter, the $\Gamma_{1}$ term in the above formula breaks time-reversal symmetry. This property reflects that the leading-order contribution to the coupling between Majoranas at the same end $\left(i \gamma_{j} \tilde{\gamma}_{j}\right)$ should come from the second-order perturbation in the tunneling process. Actually, the direct coupling between $\gamma_{j}$ and $\tilde{\gamma}_{j}$ 
does not experience the phase difference across the junction and should preserve time-reversal symmetry. The reason is because a uniform pairing phase in one end of the junction can be removed by a constant gauge transformation. Therefore, the coupling between Majorana fermions at the same end can only be induced by electron tunneling across the junction, and the minimum requirement is to consider the second-order tunneling process. Furthermore, the system restores time-reversal symmetry at $\phi=m \pi$, which explains why the $\Gamma_{1}$ term is proportional to $\sin \phi$, and has $2 \pi$ periodicity.

\section{b. Josephson current}

The Hamiltonian (A39) can be block diagonalized by a constant transformation in the Majorana bases that $\gamma_{1}^{\prime}=\gamma_{L}+\tilde{\gamma}_{R}, \gamma_{2}^{\prime}=\gamma_{R}+\tilde{\gamma}_{L}$, and $\tilde{\gamma}_{1,2}^{\prime}=\mathcal{T} \gamma_{1,2}^{\prime} \mathcal{T}^{-1}$, which sends $H_{\text {eff }}$ to be $H_{\text {eff }}=i\left(\Gamma_{0} \cos \phi / 2+\Gamma_{1} \sin \phi\right) \gamma_{1}^{\prime} \gamma_{2}^{\prime}-$ $i\left(\Gamma_{0} \cos \phi / 2-\Gamma_{1} \sin \phi\right) \tilde{\gamma}_{1}^{\prime} \tilde{\gamma}_{2}^{\prime}$. The Andreev bound-state spectra are obtained straightforwardly by $E_{n_{f^{\prime}}, \tilde{n}_{f^{\prime}}}(\phi)=$ $\left(\Gamma_{0} \cos \phi / 2+\Gamma_{1} \sin \phi\right)\left(2 n_{f^{\prime}}-1\right)+\left(\Gamma_{0} \cos \phi / 2-\Gamma_{1} \sin \phi\right)$ $\left(2 n_{\tilde{f}^{\prime}}-1\right)$, which are doubly degenerate at $\phi=m \pi$, reflecting the time-reversal symmetry at these points. Here, $n_{f^{\prime}, \tilde{f}^{\prime}}$ are complex fermion-number operators for the $f^{\prime}$ and $\tilde{f}^{\prime}$ modes, respectively. The Josephson current then reads

$$
\begin{aligned}
J_{\phi}= & \left(\frac{e \Gamma_{0}}{2 \hbar} \sin \frac{\phi}{2}+\frac{e \Gamma_{1}}{2 \hbar} \cos \phi\right)\left(2 n_{f^{\prime}}-1\right) \\
& +\left(\frac{e \Gamma_{0}}{2 \hbar} \sin \frac{\phi}{2}-\frac{e \Gamma_{1}}{2 \hbar} \cos \phi\right)\left(2 n_{\tilde{f}^{\prime}}-1\right) .
\end{aligned}
$$

From Eq. (A40), we find that for the even-parity states $(|0 \tilde{0}\rangle$ and $|1 \tilde{1}\rangle)$, the Josephson currents $J_{\phi}^{\text {even }}=$ $\pm(e / \hbar) \Gamma_{0} \sin \frac{\phi}{2}$, which are of $4 \pi$ periodicity, while for the odd-parity states $(|0 \tilde{1}\rangle$ and $|1 \tilde{0}\rangle), \quad J_{\phi}^{\text {odd }}=$ $\pm(e / \hbar) \Gamma_{1} \cos \phi$ exhibit $2 \pi$ periodicity. The difference in the periodicity reflects different mechanisms for $J_{\phi}^{\text {even, odd }}$. The currents $J_{\phi}^{\text {even }}$ are contributed from the $\Gamma_{0}$ term in the effective coupling Hamiltonian, which is due to the direct coupling between Majorana modes at different ends of the junction. Therefore, the currents $J_{\phi}^{\text {even }}$ are a consequence of the single-electron tunneling process and have $4 \pi$ periodicity. On the other hand, as contributed from the $\Gamma_{1}$ term, the Josephson currents $J_{\phi}^{\text {odd }}$ result from the second-order tunneling process, which corresponds to the Cooper-pair tunneling, therefore being of $2 \pi$ periodicity. Furthermore, the currents $J_{\phi}^{\text {odd }}$ are nonzero even for $\phi=0$, which reflects the fact that the odd-parity states violate the time-reversal symmetry that even $H_{\text {eff }}$ preserves at $\phi=m \pi$.

The $4 \pi$ periodicity of the Josephson currents for evenparity states can also be understood in the following way.
When the phase difference across the junction advances $2 \pi$, the Cooper-pair wave function changes $2 \pi$ across the junction, while for single-electron operators, the phase varies only $\pi$. Therefore, the coupling coefficients also change $\pi$ phase and thus reverse sign, leading to the $4 \pi$ periodicity of the direct coupling term. The generality of this argument implies that the $4 \pi$ periodicity of $J_{\phi}^{\text {even }}$ is stable against the disorder scattering without breaking timereversal symmetry. On the other hand, for odd-parity states, the twofold degeneracy at $\phi=m \pi$ is protected by timereversal symmetry, which shows that the qualitative properties of the Josephson currents $J_{\phi}^{\text {odd }}$ are also stable against the TRI disorder scattering. The numerical results are shown in Fig. 9.

With the above results, we can have different strategies in the experiment to distinguish $J_{\phi}^{\text {even }}$ and $J_{\phi}^{\text {odd }}$. For instance, one can measure the periodicity of the Josephson currents or measure the currents at $\phi=\pi / 2$, where $J_{\phi}^{\text {even }}=$ $\pm(e / \sqrt{2} \hbar) \Gamma_{0}$ and $J_{\phi}^{\text {odd }}=0$. Furthermore, the two qubit states of the same total parity are distinguished by current directions. The qualitative difference in the Josephson measurements provides direct detection of the four topological qubit states.

[1] G. Moore and N. Read, Nonabelions in the Fractional Quantum Hall Effect, Nucl. Phys. B360, 362 (1991).

[2] N. Read and D. Green, Paired States of Fermions in Two Dimensions with Breaking of Parity and Time-Reversal Symmetries and the Fractional Quantum Hall Effect, Phys. Rev. B 61, 10267 (2000).

[3] D. A. Ivanov, Non-Abelian Statistics of Half-Quantum Vortices in p-Wave Superconductors, Phys. Rev. Lett. 86, 268 (2001).

[4] A. Y. Kitaev, Unpaired Majorana Fermions in Quantum Wires, Phys. Usp. 44, 131 (2001).

[5] A. Kitaev, Fault-Tolerant Quantum Computation by Anyons, Ann. Phys. (Amsterdam) 303, 2 (2003).

[6] S. Das Sarma, M. Freedman, and C. Nayak, Topologically Protected Qubits from a Possible Non-Abelian Fractional Quantum Hall State, Phys. Rev. Lett. 94, 166802 (2005).

[7] C. Nayak, S. H. Simon, A. Stern, M. Freedman, and S. Das Sarma, Non-Abelian Anyons and Topological Quantum Computation, Rev. Mod. Phys. 80, 1083 (2008).

[8] L. Fu and C. L. Kane, Superconducting Proximity Effect and Majorana Fermions at the Surface of a Topological Insulator, Phys. Rev. Lett. 100, 096407 (2008).

[9] F. Wilczek, Majorana Returns, Nat. Phys. 5, 614 (2009).

[10] J. D. Sau, R. M. Lutchyn, S. Tewari, and S. Das Sarma, Generic New Platform for Topological Quantum Computation Using Semiconductor Heterostructures, Phys. Rev. Lett. 104, 040502 (2010).

[11] J. Alicea, Majorana Fermions in a Tunable Semiconductor Device, Phys. Rev. B 81, 125318 (2010).

[12] R. M. Lutchyn, J. D. Sau, and S. Das Sarma, Majorana Fermions and a Topological Phase Transition in 
Semiconductor-Superconductor Heterostructures, Phys. Rev. Lett. 105, 077001 (2010).

[13] Y. Oreg, G. Refael, and F. von Oppen, Helical Liquids and Majorana Bound States in Quantum Wires, Phys. Rev. Lett. 105, 177002 (2010).

[14] A. C. Potter and P. A. Lee, Multichannel Generalization of Kitaev's Majorana End States and a Practical Route to Realize Them in Thin Films, Phys. Rev. Lett. 105, 227003 (2010).

[15] J. Alicea, Y. Oreg, G. Refael, F. von Oppen, and M. P. A. Fisher, Non-Abelian Statistics and Topological Quantum Information Processing in 1D Wire Networks, Nat. Phys. 7, 412 (2011).

[16] A. Cook and M. Franz, Majorana Fermions in a Topological-Insulator Nanowire Proximity-Coupled to an s-Wave Superconductor, Phys. Rev. B 84, 201105(R) (2011).

[17] K. T. Law, P. A. Lee, and T. K. Ng, Majorana Fermion Induced Resonant Andreev Reflection, Phys. Rev. Lett. 103, 237001 (2009).

[18] K. Flensberg, Tunneling Characteristics of a Chain of Majorana Bound States, Phys. Rev. B 82, 180516 (2010).

[19] X.-J. Liu, Andreev Bound States in a One-Dimensional Topological Superconductor, Phys. Rev. Lett. 109, 106404 (2012).

[20] V. Mourik, K. Zuo, S. M. Frolov, S. R. Plissard, E. P. A. M. Bakkers, and L. P. Kouwenhoven Signatures of Majorana Fermions in Hybrid Superconductor-Semiconductor Nanowire Devices, Science 336, 1003 (2012).

[21] M. T. Deng, C. L. Yu, G. Y. Huang, M. Larsson, P. Caroff, and H. Q. Xu, Observation of Majorana Fermions in a $\mathrm{Nb}$-InSb Nanowire-Nb Hybrid Quantum Device, Nano Lett. 12, 6414 (2012).

[22] A. Das, Y. Ronen, Y. Most, Y. Oreg, M. Heiblum, and H. Shtrikman, Zero-Bias Peaks and Splitting in an Al-InAs Nanowire Topological Superconductor as a Signature of Majorana Fermions, Nat. Phys. 8, 887 (2012).

[23] A. P. Schnyder, S. Ryu, A. Furusaki, and A. W. W. Ludwig, Topological Insulators and Superconductors: Ten-fold Way and Dimensional Hierarchy, Phys. Rev. B 78, 195125 (2008).

[24] X.-L. Qi, T. L. Hughes, S. Raghu, and S.-C. Zhang, Time-Reversal-Invariant Topological Superconductors and Superfluids in Two and Three Dimensions, Phys. Rev. Lett. 102, 187001 (2009).

[25] J. C. Y. Teo and C. L. Kane, Topological Defects and Gapless Modes in Insulators and Superconductors, Phys. Rev. B 82, 115120 (2010).

[26] A. P. Schnyder, P. M. R. Brydon, D. Manske, and C. Timm, Andreev Spectroscopy and Surface Density of States for a Three-Dimensional Time-Reversal Invariant Topological Superconductor, Phys. Rev. B 82, 184508 (2010).

[27] C. W. J. Beenakker, J. P. Dahlhaus, M. Wimmer, and A. R. Akhmerov, Random-Matrix Theory of Andreev Reflection from a Topological Superconductor, Phys. Rev. B 83, 085413 (2011).

[28] S. Deng, L. Viola, and G. Ortiz, Majorana Modes in Time-Reversal Invariant s-Wave Topological Superconductors, Phys. Rev. Lett. 108, 036803 (2012).
[29] S. Nakosai, Y. Tanaka, and N. Nagaosa, Topological Superconductivity in Bilayer Rashba System, Phys. Rev. Lett. 108, 147003 (2012).

[30] C. L. M. Wong and K. T. Law, Realizing DIII Class Topological Superconductors Using $d_{x^{2}-y^{2}}$-Wave Superconductors, Phys. Rev. B 86, 184516 (2012).

[31] F. Zhang, C. L. Kane, and E. J. Mele, Time Reversal Invariant Topological Superconductivity and Majorana Kramers Pairs, Phys. Rev. Lett. 111, 056402 (2013).

[32] S. Nakosai, J. C. Budich, Y. Tanaka, B. Trauzettel, and N. Nagaosa, Majorana Bound States and Non-local Spin Correlations in a Quantum Wire on an Unconventional Superconductor, Phys. Rev. Lett. 110, 117002 (2013).

[33] A. Keselman, L. Fu, A. Stern, and E. Berg, Inducing Time Reversal Invariant Topological Superconductivity and Fermion Parity Pumping in Quantum Wires, Phys. Rev. Lett. 111, 116402 (2013).

[34] E. Gaidamauskas, J. Paaske, and K. Flensberg, Majorana Bound States in Two-Channel Time-Reversal-Symmetric Nanowire Systems, Phys. Rev. Lett. 112, 126402 (2014).

[35] A. Haim, A. Keselman, E. Berg, and Y. Oreg, Time-Reversal Invariant Topological Superconductivity Induced by Repulsive Interactions in Quantum Wires, arXiv:1310.4525.

[36] F. Zhang and C. L. Kane, Anomalous Topological Pumps and Fractional Josephson Effects, arXiv:1310.5281.

[37] E. Dumitrescu, J. D. Sau, and S. Tewari, Magnetic Field Response and Chiral Symmetry of Time Reversal Invariant Topological Superconductors, arXiv:1310.7938.

[38] J. Klinovaja and D. Loss, Time-Reversal Invariant Parafermions in Interacting Rashba Nanowires, arXiv: 1312.1998.

[39] E. Bauer, G. Hilscher, H. Michor, Ch. Paul, E. Scheidt, A. Gribanov, Yu. Seropegin, H. Noël, M. Sigrist, and P. Rogl Heavy Fermion Superconductivity and Magnetic Order in Noncentrosymmetric $\mathrm{CePt}_{3}$ Si, Phys. Rev. Lett. 92, 027003 (2004).

[40] M. Sato and S. Fujimoto, Topological Phases of Noncentrosymmetric Superconductors: Edge States, Majorana Fermions, and Non-Abelian Statistics, Phys. Rev. B 79, 094504 (2009).

[41] I. Turek, V. Drchal, J. Kudrnovsky, M. Sob, and P. Weinberger, Electronic Structure of Disordered Alloys, Surfaces and Interfaces (Kluwer, Boston, 1997).

[42] D. Rainis and D. Loss, Majorana Qubit Decoherence by Quasiparticle Poisoning, Phys. Rev. B 85, 174533 (2012).

[43] See Supplemental Material at http://link.aps.org/ supplemental/10.1103/PhysRevX.4.021018 for more details of the braiding statistics.

[44] X.-J. Liu and A. M. Lobos, Manipulating Majorana Fermions in Quantum Nanowires with Broken Inversion Symmetry, Phys. Rev. B 87, 060504(R) (2013).

[45] D. Sticlet, C. Bena, and P. Simon, Josephson Effect in Superconducting Wires Supporting Multiple Majorana Edge States, Phys. Rev. B 87, 104509 (2013). 